\title{
Light bending from eikonal in worldline quantum field theory
}

\section{Fiorenzo Bastianelli, Francesco Comberiati and Leonardo de la Cruz}

\author{
Dipartimento di Fisica e Astronomia "Augusto Righi", Università di Bologna, \\ and INFN Sezione di Bologna, \\ via Irnerio 46, I-40126 Bologna, Italy \\ E-mail: fiorenzo.bastianelli@bo.infn.it, \\ francesco.comberiat2@unibo.it, leonardo.delacruz@unibo.it
}

ABSTRACT: Using the worldline quantum field theory (WQFT) formalism for classical scattering, we study the deflection of light by a heavy massive spinless/spinning object. WQFT requires the use of the worldline dressed propagator of a photon in a gravitational background, which we construct from first principles. The action required to set up the worldline path integral is constructed using auxiliary variables, which describe dynamically the spin degrees of freedom of the photon and take care of path ordering. We test the fully regulated path integral by recovering the photon-photon-graviton vertex. With the dressed propagator at hand, we follow the WQFT procedure by setting up the partition function and deriving the Feynman rules which can be used to evaluate it perturbatively. These rules depend on the auxiliary variables. The latter ultimately do not contribute in the geometric-optics regime, which realizes the equivalence between the scattering of a photon and a massive scalar with that of a massless and a massive scalar. Then, the calculation of the eikonal phase and the deflection angle simplifies considerably. Using the eikonal phase defined in terms of the partition function, we calculate explicitly the deflection angle at NLO in the spinless case, and at LO in the spinning case up to quadratic order in spin.

Keywords: Black Holes, Classical Theories of Gravity, Scattering Amplitudes

ArXiv EPrint: 2112.05013 


\section{Contents}

1 Introduction 1

2 Review 3

2.1 The gravitationally dressed scalar propagator 3

2.2 Relation to scattering amplitudes 5

2.3 Worldline Feynman rules 6

3 Photons and WQFT $\quad 8$

3.1 Derivation of the gravitationally dressed photon propagator 8

$\begin{array}{lll}3.1 .1 & \text { Examples } & 10\end{array}$

$\begin{array}{lll}3.2 & \text { From dressed propagators to amplitudes } & 12\end{array}$

$\begin{array}{ll}\text { 3.3 Partition function and derivation of Feynman rules } & 14\end{array}$

3.4 Definition of the eikonal phase and deflection angle 16

$\begin{array}{ll}3.4 .1 \text { Example } & 17\end{array}$

$\begin{array}{lll}\text { 3.5 Massless limit vs photon worldline in WQFT } & 17\end{array}$

4 Calculation of deflection angles $\quad 19$

$\begin{array}{ll}4.1 \text { The spinless case } & 19\end{array}$

$\begin{array}{ll}\text { 4.1.1 Leading order } & 19\end{array}$

$\begin{array}{lll}4.1 .2 & \text { NLO } & 20\end{array}$

$\begin{array}{lll}4.2 & \text { Spinning massive particle } & 22\end{array}$

5 Conclusions $\quad 24$

$\begin{array}{ll}\text { A Next to leading order photon impulse } & 25\end{array}$

\section{Introduction}

The worldline quantum field theory (WQFT) description of classical scattering is a perturbative path integral formalism which simplifies the classical limit procedure of scattering amplitudes in gravity [1]. WQFT is based on a relation between elastic (or inelastic) scattering amplitudes in the absence of matter loops and a worldline path integral representation of the dressed Feynman propagator. ${ }^{1}$ The relation between the $S$-matrix, and dressed propagators requires a procedure to put the latter on-shell after having removed the external legs. The prescription for obtaining such propagator was pioneered by Fradkin long time ago [10] and applied in ref. [11] to study high energy scattering in gravity.

\footnotetext{
${ }^{1}$ Roughly speaking, in perturbation theory, a dressed propagator represents a resummation of tree-level Feynman diagrams of a particle propagating in a background (see figure 1). Dressed propagators have been developed in a worldline representation for a variety of models, see e.g. [2-9].
} 
Once the worldline path integral is under control and the correspondence to the $S$-matrix made explicit, expectation values can be computed from a partition function expressed as a worldline path integral. One can then derive Feynman rules of the theory which allow the calculation of these expectation values directly. This is the WQFT approach to classical scattering observables. WQFT shares some similarities with the Effective Field Theory (EFT) approach to gravitational dynamics [12-14] with the important difference that in WQFT worldline degrees of freedom are also quantized. The WQFT formalism has been further developed to describe Bremsstrahlung [15], spinning black holes [16, 17] and colored massive particles [18].

The direct relation between the WQFT formalism and on-shell scattering amplitudes motivate us to find a similar treatment of the classical scattering of light in the presence of a scalar/spinning object. ${ }^{2}$ Treating quantum gravity as an EFT [20-22] it was recently found that there are small quantum corrections that differentiate the scattering of a massless particle from that of light off a heavy mass object [23-25], thus testing the equivalence principle [26]. Since these differences are absent in the classical limit, one can recover deflection angles by taking the massless limit of the scattering of massive particles, see e.g. ref. [27]. In particular, as studied a long time ago by Amati, Ciafaloni and Veneziano [28], one can employ the eikonal phase to extract classical deflection angles in gravity through differentiation. More recently, this approach has received a renewed interest [29-46]. Another route to the problem was taken in ref. [47], where the Kosower-Maybee-O'Connell (KMOC) formalism [48-50] for classical observables was generalized to describe the classical limit of massless particles. In one of the applications of ref. [47], the geometric-optics regime was used to extract the deflection angle from scattering amplitudes, since it is in this limit in which the amplitude can be related to a beam of light through the precise definition of the classical observable. As we shall see, our WQFT construction requires this exact regime to extract the deflection angle through the eikonal phase.

In this paper, we construct the appropriate WQFT to deal with photons with a first goal of realizing the equivalence between the scattering of a photon and a massive scalar with that of a massless and a massive scalar in the geometric-optics regime. The second goal is a simplified approach to the calculation of the scattering angle. Unlike the case of a matter particle propagating in a gravitational background, the dressed photon propagator depends on a matrix-valued action and therefore the worldline path integral must include a path ordering. The path ordering can be avoided by rewriting the path integral in terms of auxiliary variables at the cost of introducing additional integrals. These variables are inherently quantum - they describe the quantum polarization of the spin 1 particle but ultimately they do not play any role in our classical computation. This insight plays a key role to show the aforementioned equivalence.

Once the dressed propagator is obtained and put on-shell, we can follow the WQFT setup to derive its Feynman rules and compute the eikonal phase. The Feynman rules that we find depend in general on the auxiliary variables. Building on the insights of

\footnotetext{
${ }^{2}$ The different approaches to describe this system and their relation with the eikonal phase is nicely reviewed in ref. [19].
} 
ref. [47], we consider the geometric-optics regime, which leads to a great simplification of the calculations. As we shall see, this regime implies the vanishing of terms proportional to the spin-tensor and serves as a check of our setup. Then, the calculation of the eikonal phase and derived quantities, such as the deflection angle and the impulse, dramatically simplifies.

The rest of the paper is organized as follows: in section 2, we review the WQFT formalism and introduce our conventions. In section 3, we derive the dressed photon propagator required for the implementation of WQFT and define the eikonal phase. In section 4 we compute the eikonal phase and deflection angles at LO and NLO. Our conclusions are presented in section 5 .

\section{Review}

\subsection{The gravitationally dressed scalar propagator}

In order to introduce our notation and conventions, let us consider first the case of a single scalar massive particle of mass $m$. We will use the mostly minus signature for the Minkowski metric $\eta_{\mu \nu}=\operatorname{diag}(1,-1,-1,-1)$ and set the gravitational coupling to $\kappa^{2}=32 \pi G_{N}$, where $G_{N}$ is the Newton constant. The gravitational action is given by the usual Einstein-Hilbert action

$$
S_{\mathrm{EH}}=-\frac{2}{\kappa^{2}} \int \mathrm{d}^{4} x \sqrt{-g} R,
$$

whereas the action for the massive scalar field including a non-minimal coupling of the scalar field to the background curvature is given by

$$
S_{\mathrm{m}}=\int \mathrm{d}^{4} x \sqrt{-g}\left[g^{\mu \nu} \partial_{\mu} \varphi^{*} \partial_{\nu} \varphi+\left(\xi R-m^{2}\right) \varphi^{*} \varphi\right] .
$$

Here $\xi$ is a free dimensionless coupling. Requiring Weyl invariance in the massless case fixes this coupling to $\xi=\frac{1}{6}$ ( $\xi=\frac{d-2}{4(d-1)}$ in arbitrary dimensions), but here we shall keep it arbitrary. In order to relate scattering amplitudes and path integrals, we first rewrite the scalar propagator in an external gravitational field in a proper time representation

$$
\mathrm{i} G(x, y ; g)=\left\langle y\left|\frac{1}{\hat{H}-\mathrm{i} \epsilon}\right| x\right\rangle=\mathrm{i} \int_{0}^{\infty} \mathrm{d} T\left\langle y\left|e^{-\mathrm{i} T(\hat{H}-\mathrm{i} \epsilon)}\right| x\right\rangle,
$$

where $T$ is the Fock-Schwinger proper time. The Hamiltonian operator $\hat{H}$ corresponds to the Klein-Gordon operator fixed by the action (2.2) and is given by

$$
\hat{H}=g^{\mu \nu} \nabla_{\mu} \nabla_{\nu}+m^{2}-\xi R=\frac{1}{\sqrt{-g}} \partial_{\mu} \sqrt{-g} g^{\mu \nu} \partial_{\nu}+m^{2}-\xi R .
$$

It can be viewed as arising from a classical particle Hamiltonian obtained by setting $\partial_{\mu} \rightarrow$ $-\mathrm{i} p_{\mu}$ in the last expression, finding $H=-g^{\mu \nu} p_{\mu} p_{\nu}+m^{2}-\xi R$. The particle action in hamiltonian form can be written as

$$
S_{\mathrm{p}}=\int_{0}^{1} \mathrm{~d} \tau\left(p_{\mu} \dot{x}^{\mu}-e H\right),
$$


where $e$ is the einbein that gauges translations on the worldline, leading to a reparametrization invariant description of the worldline. The einbein reproduces the effect of the proper time $T$ upon gauge fixing $e(\tau)=T$. Then, rescaling the proper time $\tau$ to range in the interval $[0, T]$, we obtain the particle action in configuration space

$$
S_{\mathrm{p}}=\int_{0}^{T} \mathrm{~d} \tau\left[-\frac{1}{4} g_{\mu \nu} \dot{x}^{\mu} \dot{x}^{\nu}-m^{2}+\xi R\right]
$$

In order to define a path integral free of spurious UV divergences and regularization ambiguities, one must introduce auxiliary worldline ghost variables and a finite counterterm to the worldline action (2.6). Let us mention that these issues are only related to the onedimensional worldline theory, and are not related to regularization of spacetime. The case of UV divergences on the worldline can be addressed by defining the path integral measure as follows

$$
\mathcal{D} x:=D x \prod_{0<\tau<T} \sqrt{-g(x(\tau))}=D x \int D a D b D c \exp \left[-\mathrm{i} \int_{0}^{T} \mathrm{~d} \tau \frac{1}{4} g_{\mu \nu}\left(a^{\mu} a^{\nu}+b^{\mu} c^{\nu}\right)\right],
$$

where the final form contains the standard translationally invariant measures, indicated by the symbol $D$ as opposed to the symbol $\mathcal{D}$. The second equality exponentiates the determinant factor and leads to standard perturbation theory on the worldline. As regularization ambiguities play no role in the upcoming discussion, let us just mention that three options to fix such ambiguities are known, and correspond to the time slicing (TS) regularization, mode regularization (MR), and worldline dimensional regularization (DR) [51]. The appropriate counterterms in these schemes can be written as follows

$$
S_{\mathrm{CT}}=\int_{0}^{T} \mathrm{~d} \tau\left(-\frac{1}{4} R-V_{\mathrm{TS} / \mathrm{MR} / \mathrm{DR}}\right),
$$

where the additional terms $V_{\mathrm{TS} / \mathrm{MR} / \mathrm{DR}}$ are scheme dependent. ${ }^{3}$ The path integral in configuration space associated with the propagator is finally given by (see figure 1).

$$
\begin{aligned}
G(x, y ; g)= & \int_{0}^{\infty} \mathrm{d} T e^{-\mathrm{i} m^{2} T} \int_{x(0)=x}^{x(T)=y} D x \int D a D b D c \\
& \exp \left\{-\mathrm{i} \int_{0}^{T} \mathrm{~d} \tau\left[\frac{1}{4}\left(g_{\mu \nu} \dot{x}^{\mu} \dot{x}^{\nu}+a^{\mu} a^{\nu}+b^{\mu} c^{\nu}\right)+\left(\frac{1}{4}-\xi\right) R+V_{\mathrm{TS} / \mathrm{MR} / \mathrm{DR}}\right]\right\} .
\end{aligned}
$$

It can be solved in perturbation theory with standard gaussian integration $[53,54]$. In order to apply this technique, we consider the splitting of the position variable into a straight line and a quantum fluctuation $q^{\mu}$

$$
x^{\mu}(\tau)=b^{\mu}+v^{\mu} \tau+q^{\mu}(\tau) .
$$

At this point the dressed propagator represents a resummation of tree-level Feynman diagrams in which the straight line parameters $b^{\mu}$ and $u^{\mu}$ depend on boundary conditions

\footnotetext{
${ }^{3}$ They are given by $V_{\mathrm{TS}}=-1 / 4 g^{\mu \nu} \Gamma_{\mu \alpha}^{\beta} \Gamma_{\nu \beta}^{\alpha}, V_{\mathrm{MR}}=1 / 12 g^{\mu \nu} g^{\alpha \beta} g_{\rho \sigma} \Gamma_{\mu \alpha}^{\rho} \Gamma_{\nu \beta}^{\sigma}$, and $V_{\mathrm{DR}}=0$. Counterterms for supersymmetric versions of the nonlinear sigma model can be found in [52].
} 


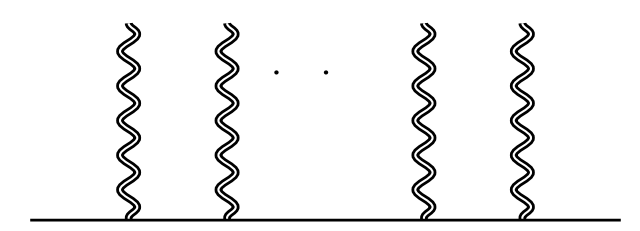

Figure 1. Dressed propagator with external massive particles off-shell.

one wishes to impose, and the quantum-fluctuation variables acquire Dirichlet boundary conditions (DBC), namely $q(0)=q(T)=0$. As we will review shortly, relating dressed propagators and scattering amplitudes in the classical limit requires to appropriately express the boundary conditions carried by the parameters of the straight line in terms of the physical parameters of the scattering.

\subsection{Relation to scattering amplitudes}

Consider the elastic scattering of two spinless massive particles of masses $m_{1}$ and $m_{2}$ in the absence of matter loops. Each massive particle is described by his own dressed propagator, which we can write as $G_{i}(x, y ; g), i=1,2$ with obvious labeling of the worldline variables and masses. The dressed propagators are connected but not amputated. In order to define the $S$-matrix we must amputate the external legs and put them on-shell. In momentum space the amputated on-shell dressed propagator reads

$$
G^{\mathrm{c}}\left(p, p^{\prime} ; g\right):=\lim _{p^{2}, p^{\prime 2} \rightarrow m^{2}} \mathrm{i}\left(p^{2}-m^{2}\right) \mathrm{i}\left(p^{\prime 2}-m^{2}\right) \int \mathrm{d}^{4} x \mathrm{~d}^{4} y e^{\mathrm{i} p \cdot x-\mathrm{i} p^{\prime} \cdot y} G(x, y ; g) .
$$

The overall effect of putting the dressed propagator on-shell amounts to eliminate the proper time integral in (2.9) and set the region of integration over $\tau$ in the action to $\tau \in(-\infty,+\infty)$, as shown in ref. [1].

Let us now consider the weak field approximation $g_{\mu \nu}=\eta_{\mu \nu}+\kappa h_{\mu \nu}$, and add to the Einstein-Hilbert action the gauge-fixing term

$$
S_{\text {gf }}=\int \mathrm{d}^{4} x\left(\partial^{\nu} h_{\mu \nu}-1 / 2 \partial_{\mu} h_{\nu}^{\nu}\right)^{2}
$$

which imposes a weighted version of the de Donder gauge $\partial^{\nu} h_{\mu \nu}=1 / 2 \partial_{\mu} h^{\nu}{ }_{\nu}$. The full action is then

$$
S_{g}=S_{\mathrm{EH}}+S_{\mathrm{gf}} .
$$

The on-shell version of the dressed propagators can then be used to relate the elastic scattering amplitude in the absence of matter loops as follows

$$
\mathcal{A}\left(p_{1} p_{2} \rightarrow p_{1}^{\prime} p_{2}^{\prime}\right)=\mathcal{N} \int \mathcal{D} g_{\mu \nu} e^{\mathrm{i} S_{g}} G_{1}^{c}\left(p_{1}, p_{1}^{\prime} ; g\right) G_{2}^{c}\left(p_{1}, p_{2}^{\prime} ; g\right)
$$

where $\mathcal{N}$ is a normalization factor. The classical limit on the right hand side is taken and understood as the usual one in QFT, i.e., considering only graviton Born diagrams (see figure 2). To avoid the proliferation of factors of $2 \pi$, we will adopt the short-hand notation

$$
\hat{\mathrm{d}}^{n} x:=\frac{\mathrm{d}^{n} x}{(2 \pi)^{n}}, \quad \hat{\delta}^{n}(x):=(2 \pi)^{n} \delta^{n}(x) .
$$




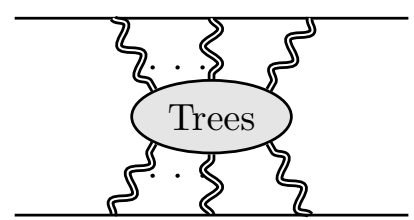

(a) elastic

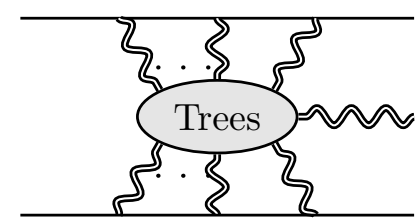

(b) inelastic

Figure 2. Diagrams resummed by the method. The blob represent trees including disconnected ones.

We define the eikonal phase as in refs. $[28,55]$ by

$$
e^{\mathrm{i} \chi}:=\frac{1}{4 m_{1} m_{2}} \int \hat{\mathrm{d}}^{d} q \hat{\delta}\left(q \cdot v_{1}\right) \hat{\delta}\left(q \cdot v_{2}\right) e^{\mathrm{i} q \cdot b} \mathcal{A}\left(p_{1} p_{2} \rightarrow p_{1}^{\prime} p_{2}^{\prime}\right),
$$

where $b:=b_{2}-b_{1}$ is the impact parameter and $q:=p_{1}^{\prime}-p_{1}=p_{2}-p_{2}^{\prime}$ is the momentum exchange. The eikonal phase and the QFT S-matrix (in the classical limit) in the worldline theory can be nicely related as [1]

$$
\mathcal{Z}_{\mathrm{WFT}}=e^{\mathrm{i} \chi}
$$

which links the free energy of the WQFT and the eikonal phase. The path integral representation of the partition function can be written as

$$
\begin{aligned}
\mathcal{Z}_{\mathrm{WFT}}:= & \mathcal{N} \int D h_{\mu \nu} \int \prod_{i=1}^{2} D x_{i} \int \prod_{i=1}^{2}\left(D a_{i} D b_{i} D c_{i}\right) e^{\mathrm{i} S_{g}} \\
& \exp \left[-\mathrm{i} \sum_{i=1}^{2} \int_{-\infty}^{\infty} \mathrm{d} \tau_{i} \frac{m_{i}}{2} g_{\mu \nu}\left(\dot{x}_{i}^{\mu} \dot{x}_{i}^{\nu}+a_{i}^{\mu} a_{i}^{\nu}+b_{i}^{\mu} c_{i}^{\nu}\right)\right],
\end{aligned}
$$

where the normalization factor $\mathcal{N}$ ensures that $\mathcal{Z}_{\mathrm{WFT}}=1$ in the free theory. Expectation values of operators $\mathcal{O}\left(h,\left\{x_{i}\right\}\right)$ are obtained as

$$
\begin{aligned}
\left\langle\mathcal{O}\left(h,\left\{x_{i}\right\}\right)\right\rangle_{\mathrm{WQFT}}= & \mathcal{Z}_{\mathrm{WFT}}^{-1} \int D h_{\mu \nu} \int \prod_{i=1}^{2} D x_{i} \int \prod_{i=1}^{2}\left(D a_{i} D b_{i} D c_{i}\right) e^{\mathrm{i} S_{g}} \mathcal{O}\left(h,\left\{x_{i}\right\}\right) \\
& \exp \left[-\mathrm{i} \sum_{i=1}^{2} \int_{-\infty}^{\infty} \mathrm{d} \tau_{i} \frac{m_{i}}{2} g_{\mu \nu}\left(\dot{x}_{i}^{\mu} \dot{x}_{i}^{\nu}+a_{i}^{\mu} a_{i}^{\nu}+b_{i}^{\mu} c_{i}^{\nu}\right)\right]
\end{aligned}
$$

\subsection{Worldline Feynman rules}

The path integral can be solved perturbatively using worldline Feynman rules which keep track of the expansion in power of the coupling $\kappa$ and take care of the Wick contractions between fields and the quantum fluctuations $q_{i}$. The role of the auxiliary ghost variables $a_{i}, b_{i}, c_{i}$ is to cancel divergences which are of quantum nature. Since we are not interested in those corrections, it is enough to summarize the Feynman rules associated with the fields $h_{\mu \nu}$ and the deflections $q^{\mu}(\tau)$. In order to make contact more naturally with onshell scattering amplitudes, we scale the worldline parameters of the particle action as $\tau_{i} \rightarrow \sigma_{i} / m_{i}$ such that the background split in configuration space reads

$$
x_{i}^{\mu}\left(\sigma_{i}\right)=b_{i}^{\mu}+p_{i}^{\mu} \sigma_{i}+q_{i}^{\mu}\left(\sigma_{i}\right),
$$


where $p_{i}^{\mu}=m_{i} v_{i}^{\mu}$. The Fourier transforms to momentum space and energy space ${ }^{4}$ for $h_{\mu \nu}(x)$ and $q^{\mu}(\sigma)$, respectively are defined by

$$
h_{\mu \nu}(x)=\int \hat{\mathrm{d}}^{4} \ell e^{-\mathrm{i} \ell \cdot x} h_{\mu \nu}(\ell), \quad q^{\mu}(\sigma)=\int \hat{\mathrm{d}} \omega e^{-\mathrm{i} \omega \sigma} q^{\mu}(\omega) .
$$

Let $P_{\mu \nu \rho \sigma}:=\left(\eta_{\mu(\rho} \eta_{\nu) \sigma}-\frac{1}{2} \eta_{\mu \nu} \eta_{\rho \sigma}\right)$, where the parenthesis of Lorentz indices denotes symmetrization with unit weight, e.g. $v_{1}^{(\mu} v_{2}^{\nu)}=\frac{1}{2}\left(v_{1}^{\mu} v_{2}^{\nu}+v_{1}^{\nu} v_{2}^{\mu}\right)$. The propagators then read

$$
q^{\mu} \stackrel{\omega}{\longrightarrow} \bullet q^{\nu}=-\mathrm{i} \frac{\eta^{\mu \nu}}{2}\left(\frac{1}{(\omega+\mathrm{i} \epsilon)^{2}}+\frac{1}{(\omega-\mathrm{i} \epsilon)^{2}}\right), h_{\mu \nu} \text { ح } h_{\rho \sigma}=\mathrm{i} \frac{P_{\mu \nu \rho \sigma}}{k^{2}+\mathrm{i} \epsilon},
$$

i.e., we use time-symmetric propagator for worldlines and Feynman propagators for gravitons. The choice of $\mathrm{i} \epsilon$ prescription determines the precise interpretation of the background parameters. Let us briefly summarize the findings of ref. [1] (see also ref. [16]). With retarded (advanced) propagators the background parameters $b^{\mu}, v^{\mu}$ are associated with their undeflected trajectories when $\tau \rightarrow-\infty(\tau \rightarrow+\infty)$. The time-symmetric prescription averages between these options

$$
v^{\mu}=\frac{1}{2}\left(v_{-\infty}^{\mu}+v_{+\infty}^{\mu}\right)+\mathcal{O}\left(G_{N}^{2}\right), \quad b^{\mu}=\frac{1}{2}\left(b_{-\infty}^{\mu}+b_{+\infty}^{\mu}\right)+\mathcal{O}\left(G_{N}^{2}\right),
$$

which are the background parameters we will use in our calculations of scattering angles from the eikonal phase. Therefore, the relation of the momenta of massive particles $p_{i}^{\mu}=$ $m_{i} v_{i}$ is associated with the average four velocities. At the maximum order we consider $(2 \mathrm{PM})$ in this paper, the difference between the far past or future background parameters are of order $\mathcal{O}\left(G_{N}\right)$ and it follows that $b_{ \pm \infty}^{\mu}=b^{\mu}+\mathcal{O}\left(G_{N}\right)$. Therefore it will be not necessary to distinguish between $\left|b_{-\infty}\right|$ and $|b|$. The interactions are then described by the rules

$$
=-\mathrm{i} \frac{\kappa}{2} e^{\mathrm{i} k \cdot b \hat{\delta}(k \cdot p) p^{\mu} p^{\nu},}
$$

Additional Feynman rules with more fluctuations along the worldline arise from higher order terms of the perturbative expansion. They are required for the calculation of the impulse and radiation. The interested reader can check ref. [1].

\footnotetext{
${ }^{4}$ Given the rescaling we performed in the worldline action, the worldline parameter $[\sigma] \sim L^{2}$ then implies that $[\omega] \sim L^{-2}$ to keep dimensionless the plane wave exponential in Fourier transforms.
} 


\section{Photons and WQFT}

Our goal is to derive the partition function that describes scattering of photons and massive scalar/spinning particles in a gravitational background. To achieve this goal, we derive the photon propagator dressed by gravitons and its worldline path integral representation. Then, we can use this expression to express the amplitude in terms of path integrals as in eq. (2.14) where one of the dressed propagators is associated with a photon.

\subsection{Derivation of the gravitationally dressed photon propagator}

Let us now add to the Einstein-Hilbert action, the action of Maxwell theory minimally coupled to gravity

$$
S_{\gamma}=-\frac{1}{4} \int \mathrm{d}^{4} x \sqrt{-g} g^{\mu \alpha} g^{\nu \beta} F_{\mu \nu} F_{\alpha \beta} .
$$

Its gauge symmetry, $\delta A_{\mu}(x)=\partial_{\mu} \alpha(x), \delta g_{\mu \nu}(x)=0$, can be covariantly gauge-fixed using standard BRST methods. The procedure is as follows. One replaces the gauge parameter $\alpha(x)$ by the anticommuting ghost $c(x)$, and from the gauge algebra obtains the anticommuting BRST variation $s$ (the so-called Slavnov variation). It is required to be nilpotent, and is then extended to the non-minimal fields needed for gauge fixing, the antighost $\bar{c}(x)$ and auxiliary $B(x)$, which are Graßmann odd and even, respectively. The BRST symmetry is

$$
s A_{\mu}=\partial_{\mu} c, \quad s c=0, \quad s \bar{c}=B, \quad s B=0,
$$

and can be easily verified to be nilpotent $\left(s^{2}=0\right)$. It is used to obtain the gauge-fixed total action by adding to the lagrangian contained in (3.1) the manifestly BRST invariant term $s \Psi_{\xi}$, where $\Psi_{\xi}=\sqrt{-g} \bar{c}\left(\nabla^{\mu} A_{\mu}+\frac{\xi}{2} B\right)$ is the gauge fermion chosen to produce a $R_{\xi}$ gauge in curved space. The fields $c, \bar{c}$ and $B$ are all taken to be scalars under change of coordinates, so to keep covariance manifest. One finds

$$
s \Psi_{\xi}=\sqrt{-g}\left(B \nabla^{\mu} A_{\mu}+\frac{\xi}{2} B^{2}-\bar{c} \nabla^{\mu} \partial_{\mu} c\right) \sim \sqrt{-g}\left(-\frac{1}{2 \xi}\left(\nabla^{\mu} A_{\mu}\right)^{2}-\bar{c} \nabla^{\mu} \partial_{\mu} c\right),
$$

where in the last step the auxiliary field $B$ has been eliminated by its own algebraic equations of motion. We choose the value $\xi=1$, that implements the Feynman gauge. The total gauge-fixed BRST invariant action, $S_{\text {tot }}=S_{\gamma}+\int \mathrm{d}^{4} x s \Psi_{\xi}$, contains a ghost action that we disregard (at tree-level ghosts do not contribute) and terms that identify the photon propagator reads

$$
S_{\gamma, \text { gf }}=\int \mathrm{d}^{4} x \sqrt{-g}\left[-\frac{1}{4} F_{\mu \nu} F^{\mu \nu}-\frac{1}{2}\left(\nabla^{\mu} A_{\mu}\right)^{2}\right]=\int \mathrm{d}^{4} x \sqrt{-g}\left[\frac{1}{2} A^{\mu} \hat{H}_{\mu}{ }^{\nu} A_{\nu}\right],
$$

where the second form is obtained by performing partial integrations. It produces the second order differential operator

$$
\hat{H}_{\mu}{ }^{\nu}=\delta_{\mu}{ }^{\nu} \nabla^{2}-R_{\mu}{ }^{\nu},
$$

whose inverse gives the photon propagator in a curved background, i.e. the dressed propagator of our interest. The operator $\hat{H}_{\mu}{ }^{\nu}$ may be interpreted as a first-quantized Hamiltonian. 
For that purpose, we find it convenient to use flat indices by introducing a vielbein $e_{\mu}^{a}(x)$, so the metric is given by $g_{\mu \nu}(x)=\eta_{a b} e_{\mu}^{a}(x) e_{\nu}^{b}(x)$. This allows to present the Hamiltonian as

$$
\hat{H}_{a}{ }^{b}=\delta_{a}{ }^{b} \nabla^{2}-R_{a}{ }^{b},
$$

where the covariant derivative contains also the spin connection $\omega_{\mu a}^{b}(x)$, as it acts on vectors $A_{a}(x)=e_{a}^{\mu}(x) A_{\mu}(x)$ with flat indices, so the covariant derivative is $\nabla_{\mu} A_{a}=\partial_{\mu} A_{a}+$ $\omega_{\mu a}^{b} A_{b}$. On a more general Lorentz tensor, the covariant derivative takes the form $\nabla_{\mu}=$ $\partial_{\mu}-\frac{1}{2} \omega_{\mu}^{a b} S_{a b}$, with $S_{a b}$ the Lorentz generators in the representation of the tensor.

Then, as in section 2.1, we find that the Hamiltonian (3.6) can be reproduced by quantization of a relativistic particle now with matrix-valued action given by

$$
\left(S_{\mathrm{p}}[x ; g]\right)_{a}{ }^{b}=\int_{0}^{1} \mathrm{~d} \tau\left(-\frac{1}{4 T} g_{\mu \nu} \dot{x}^{\mu} \dot{x}^{\nu} \delta_{a}{ }^{b}-\frac{1}{2} \dot{x}^{\mu} \omega_{\mu}^{c d}\left(S_{c d}\right)_{a}{ }^{b}+T R_{a}{ }^{b}-\frac{1}{4} T R \delta_{a}{ }^{b}\right),
$$

which contains the Lorentz generator in the spin-1 representation

$$
\left(S_{c d}\right)_{a}^{b}=\mathrm{i}\left(\eta_{c a} \delta_{d}^{b}-\eta_{d a} \delta_{c}^{b}\right)
$$

We have already gauge-fixed the einbein $e(\tau)$, required to have a reparametrization invariant description of the worldline, to the Fock-Schwinger proper time $T$, used in heat kernel methods to exponentiate the inverse of a differential operator as in (2.3). The last term in the action is the counter-term for regularization (DR) of the path integral, that we have already anticipated. It is the same one that appears in the scalar particle case, see eq. (2.8).

The particle action leads to a quantum mechanical transition amplitude, represented by the following worldline path integral

$$
D_{a}{ }^{b}\left(x_{0}, y_{0} ; g\right)=\int_{0}^{\infty} \mathrm{d} T\left\langle y_{0}, a\left|e^{-\mathrm{i} T \hat{H}}\right| x_{0}, b\right\rangle=\int_{0}^{\infty} \mathrm{d} T \int_{x(0)=x_{0}}^{x(1)=y_{0}} \mathcal{D} x \mathrm{~T}\left(e^{\mathrm{i} S_{\mathrm{p}}[x ; g]}\right)_{a}{ }^{b},
$$

where $\mathrm{T}$ denotes path ordering. It furnishes a representation of the photon propagator in a graviton background. The path ordering prescription in the path integral generates the correct gauge transformation of the quantum mechanical transition amplitude, which behaves as a bi-tensor under the local Lorentz gauge group that acts of the flat indices (see ref. [56] for more details on this worldline model and the counterterm).

For perturbative calculations, we find it useful to introduce auxiliary bosonic variables $Q_{a}$ and $\bar{Q}^{a}$, that allow to treat dynamically the spin degrees of freedom of the photon while encoding simultaneously the time-ordering prescription. ${ }^{5}$ The quantization of these variables produces an enlarged Hilbert space, which must be reduced to the appropriate one corresponding to the spin of the photon by a projection mechanism. The latter is achieved by coupling the new variables to a U(1) worldline gauge field, with an additional ChernSimons coupling set to achieve projection precisely onto the required Hilbert subspace. At the end, the gauge fixing of the additional U(1) gauge field leaves just an integration over an angle $\phi$, a modulus that describes gauge invariant configurations. In the following, we

\footnotetext{
${ }^{5}$ The same construction can be done using fermionic variables as well.
} 
use the variable $z=e^{\mathrm{i} \phi}$, and the modular integration is obtained by integrating $z$ over the unit circle of the complex plane. This construction has been exemplified in [57-59] and [4] for worldlines with the topologies of a loop and an interval, respectively (see also [60] for a pedagogical description in the case of the bi-adjoint particle). Eventually, the net effect of introducing the auxiliary variables $Q_{a}$ and $\bar{Q}^{a}$ with their $\mathrm{U}(1)$ coupling is to bring the dressed propagator of the photon into the form

$$
D\left(x_{0}, y_{0}, u, \bar{u} ; g\right)=\oint \frac{\mathrm{d} z}{2 \pi \mathrm{i}} \frac{e^{z \bar{u} \cdot u}}{z^{2}} \int_{0}^{\infty} \mathrm{d} T \int_{x(0)=x_{0}}^{x(1)=y_{0}} \mathcal{D} x \int_{\lambda(0)=0}^{\bar{\lambda}(1)=0} D \lambda D \bar{\lambda} e^{\mathrm{i} S},
$$

where the auxiliary variables have been decomposed as

$$
\bar{Q}^{a}(\tau)=z \bar{u}^{a}+\bar{\lambda}^{a}(\tau), \quad Q_{a}(\tau)=u_{a}+\lambda_{a}(\tau)
$$

with $\lambda_{a}$ and $\bar{\lambda}^{a}$ denoting the quantum fluctuations, and with the remaining classical parts $u_{a}$ and $\bar{u}^{a}$ describing the initial and final polarization of the photon depending on the modulus $z$ as indicated. It requires a worldline action that now reads

$$
S=\int_{0}^{1} \mathrm{~d} \tau\left(-\frac{1}{4 T} g_{\mu \nu} \dot{x}^{\mu} \dot{x}^{\nu}+\mathrm{i} \bar{\lambda}^{a} \dot{\lambda}_{a}-\frac{1}{2} \dot{x}^{\mu} \omega_{\mu}^{c d}\left(S_{c d}\right)_{a}{ }^{b} \bar{Q}^{a} Q_{b}+T R_{a}{ }^{b} \bar{Q}^{a} Q_{b}-\frac{3}{4} T R\right) .
$$

The splitting of the auxliliary variables into background and fluctuations is analogous to the splitting of the path $x^{\mu}(\tau)$ into a classical part plus quantum fluctuations satisfying Dirichlet boundary conditions performed earlier. Note that in ref. [61] this precise worldline model (but with fermionic auxiliary variables and treated only on a loop) appears as the one corresponding to the ghost sector of gravity covariantly quantized.

\subsubsection{Examples}

The relation between the dressed propagator and the on-shell amplitude given in section 2.2 is not the only way to obtain scattering data from the dressed propagator. Arguably the simplest way to obtain scattering data is to consider the LSZ procedure already at the level of the dressed propagator which leads to tree-level Feynman diagrams. After applying the LSZ procedure, the dressed propagator in eq. (2.9) leads to the sum of Feynman diagrams whose topology corresponds to gravitons attached to the matter line as depicted in figure 1. Similarly, the simplest cases of the dressed photon propagator are those where there is no graviton attached to the photon line and the case where a single graviton is attached to the photon line, or in other words the free photon propagator and the 3-point vertex, respectively. It is instructive to consider these cases separately to see the role of the auxiliary variables and the counterterms in the action (3.12) before going to the semiclassical matching.

Free photon propagator. The free photon propagator is obtained by switching off the interactions in (3.12) and setting $g_{\mu \nu}=\eta_{\mu \nu}$. Then, the worldline action reduces to

$$
S_{\text {free }}=\int_{0}^{1} \mathrm{~d} \tau\left(-\frac{1}{4 T} \eta_{\mu \nu}\left(\dot{x}^{\mu} \dot{x}^{\nu}+a^{\mu} a^{\nu}+b^{\mu} c^{\nu}\right)+\mathrm{i} \bar{\lambda}^{a} \dot{\lambda}_{a}\right)
$$


Expanding the coordinates as $x=x_{0}+\left(y_{0}-x_{0}\right) \tau+q(\tau)$, the free path integral on quantum fluctuations produces the measure $(4 \pi T)^{-2}$, while the classical trajectory has the simple action $S_{\mathrm{cl}}=-\left(x_{0}-y_{0}\right)^{2} / 4 T$, and we are left with

$$
D\left(x_{0}, y_{0}, u, \bar{u} ; g\right)=\oint \frac{\mathrm{d} z}{2 \pi \mathrm{i}} \frac{e^{z u \cdot \bar{u}}}{z^{2}} \int_{0}^{\infty} \frac{\mathrm{d} T}{(4 \pi T)^{2}} e^{-\mathrm{i} \frac{\left(x_{0}-y_{0}\right)^{2}}{4 T}}=\bar{u}^{\mu} \Delta_{\mu \nu}\left(x_{0}-y_{0}\right) u^{\nu},
$$

which leads to the causal photon propagator in Feynman gauge once stripping off the auxiliary variables, i.e.,

$$
\Delta_{\mu \nu}(x-y)=\eta_{\mu \nu} \int_{0}^{\infty} \frac{\mathrm{d} T}{(4 \pi T)^{2}} e^{-\mathrm{i} \frac{(x-y)^{2}}{4 T}}=\int \hat{\mathrm{d}}^{4} p \frac{\mathrm{i} \eta_{\mu \nu}}{p^{2}} e^{\mathrm{i} p \cdot(x-y)} .
$$

Photon-photon-graviton vertex. Let us now consider the more interesting case of a single graviton. In this case, we have to take into account quantum fluctuations, which we implement by performing a background expansion of the metric tensor and the configuration space variables as

$$
g_{\mu \nu}(x(\tau))=\eta_{\mu \nu}+\kappa \varepsilon_{\mu \nu}(\ell) e^{\mathrm{i} \ell \cdot x(\tau)}, \quad x^{\mu}(\tau)=x_{0}^{\mu}+\left(y_{0}-x_{0}\right)^{\mu} \tau+q^{\mu}(\tau)
$$

with the quantum fluctuations $q^{\mu}(\tau)$ acquiring DBC, i.e. $q^{\mu}(0)=q^{\mu}(1)=0$. Notice that we have Fourier expanded the graviton field as a unique plane wave to insert just one graviton in a photon line with momentum $\ell$. The contributions to the three-point amplitude arise from the interactions which we organize as follows:

$$
\begin{aligned}
S_{\text {kin }} & :=\int_{0}^{1} \mathrm{~d} \tau\left(-\frac{1}{4 T} h_{\mu \nu}(x)\left(\dot{x}^{\mu} \dot{x}^{\nu}+a^{\mu} a^{\nu}+b^{\mu} c^{\nu}\right)\right), \\
S_{\text {spin }} & :=-\frac{1}{2} \int_{0}^{1} \mathrm{~d} \tau \dot{x}^{\mu} \omega_{\mu}^{c d}\left(S_{c d}\right)_{a}{ }^{b} \bar{Q}^{a} Q_{b}, \\
S_{\text {Ric }} & :=T \int_{0}^{1} \mathrm{~d} \tau R_{a}{ }^{b} \bar{Q}^{a} Q_{b}, \\
S_{\text {ct }} & :=-\frac{3}{4} T \int_{0}^{1} \mathrm{~d} \tau R .
\end{aligned}
$$

Except for $S_{\text {kin }}$ the remaining interactions have to be background-field expanded up to $\mathcal{O}(\kappa)$ in order to give contributions to the three-point amplitude. Next, we rewrite the dressed propagator in momentum space introducing the external momenta of the photons $p_{1}, p_{2}$, so that

$$
\begin{aligned}
& \widetilde{D}\left(p_{1}, p_{2}, u, \bar{u}\right)=\int \mathrm{d}^{d} x_{0} \mathrm{~d}^{d} y_{0} e^{\mathrm{i} p_{1} \cdot x_{0}} e^{-\mathrm{i} p_{2} \cdot y_{0}} D\left(x_{0}, y_{0}, u, \bar{u} ; g\right) \\
& \quad=\hat{\delta}\left(p_{1}-p_{2}+\ell\right) \oint \frac{\mathrm{d} z}{2 \pi \mathrm{i}} \frac{e^{z u \cdot \bar{u}}}{z^{2}} \int_{0}^{\infty} \frac{\mathrm{d} T}{(4 \pi T)^{\frac{d}{2}}} e^{-\frac{\mathrm{i} \xi^{2}}{4 T}} \int \mathrm{d}^{d} \xi e^{-\mathrm{i} \xi \cdot p_{2}}\left\langle e^{\mathrm{i}\left(S_{\mathrm{kin}}+S_{\mathrm{spin}}+S_{\mathrm{Ric}}+S_{\mathrm{ct}}\right)}\right\rangle
\end{aligned}
$$

where the expectation value evaluated on the free theory, i.e., w.r.t. the action (3.13). In the second equality we have performed the change of variables $2 x_{+}=y_{0}+x_{0}, \xi=$ 
$y_{0}-x_{0}$ to factor out the momentum conservation delta function. We can now perform the perturbative expansion of the path integral. ${ }^{6}$ After evaluating all the contributions from each term we strip-off the auxiliary variables as in the free photon propagator example and amputate the external legs (see also ref. [4] for a similar treatment in the case of Yang-Mills).

We list here the final results of the on-shell procedure for each contribution from the perturbative expansion. For the kinetic term (including the regulating ghost interaction) and the spin connection vertex, we obtain

$$
A_{\mathrm{kin}}^{\alpha \beta}=-\frac{\mathrm{i} \kappa}{4}\left(p_{1}+p_{2}\right)^{\mu}\left(p_{1}+p_{2}\right)^{\nu} \varepsilon_{\mu \nu}(\ell) \eta^{\alpha \beta}, \quad A_{\mathrm{spin}}^{\alpha \beta}=\frac{\mathrm{i} \kappa}{2}\left(p_{1}^{\beta} \eta^{\alpha(\mu} p_{2}^{\nu)}+p_{2}^{\alpha} \eta^{\beta(\mu} p_{1}^{\nu)}\right) \varepsilon_{\mu \nu}(\ell),
$$

respectively. The Ricci tensor vertex contribution reads

$$
\begin{aligned}
A_{\mathrm{Ric}}^{\alpha \beta}= & \frac{\mathrm{i} \kappa}{2}\left[-\eta^{\mu \nu}\left(p_{1}^{\beta} p_{2}^{\alpha}+s_{12} \eta^{\alpha \beta}\right)+2 \eta^{\alpha \beta} p_{1}^{(\mu} p_{2}^{\nu)}\right. \\
& \left.+p_{1}^{\beta} \eta^{\alpha(\mu} p_{2}^{\nu)}+p_{2}^{\alpha} \eta^{\beta(\mu} p_{1}^{\nu)}-s_{12} \eta^{\alpha(\mu} \eta^{\nu) \beta}\right] \varepsilon_{\mu \nu}(\ell),
\end{aligned}
$$

where $s_{12}=2 p_{1} \cdot p_{2}$. Finally, the counter-term vertex generates

$$
A_{\mathrm{ct}}^{\alpha \beta}=\frac{3 \mathrm{i} \kappa}{4}\left(s_{12} \eta^{\mu \nu} \eta^{\alpha \beta}-2 \eta^{\alpha \beta} p_{1}^{(\mu} p_{2}^{\nu)}\right) \varepsilon_{\mu \nu}(\ell) .
$$

In all of these contributions the free indices contract the external photon polarization vectors. In this way, adding the above terms we obtain the three point vertex

$$
\begin{aligned}
2^{\beta} 233^{\mu \nu}= & \frac{i}{2}\left(\frac{1}{2} s_{12} \eta^{\alpha \beta} \eta^{\mu \nu}-s_{12} \eta^{\alpha(\mu} \eta^{\nu) \beta}-2 \eta^{\alpha \beta} p_{1}^{(\mu} p_{2}^{\nu)}\right. \\
& \left.+2 p_{1}^{\beta} p_{2}^{(\mu} \eta^{\nu) \alpha}+2 p_{2}^{\alpha} p_{1}^{(\mu} \eta^{\nu) \beta}-\eta^{\mu \nu} p_{1}^{\beta} p_{2}^{\alpha}\right),
\end{aligned}
$$

where the momentum conservation delta function has been stripped off. Higher point examples follow a similar procedure but with a more involved structure. However it is obvious already that the dressed propagator contains much more information than required in the classical limit. Moreover, it raises the question of what is the role, if any, of the auxiliary variables in the classical limit. Remember that they were introduced only to keep track of time-ordering which is requirement forced upon us by the quantization via path integrals. The key element to answer this question is the matching between the scattering amplitude and a pair of dressed propagators which we discuss next.

\subsection{From dressed propagators to amplitudes}

Following ref. [11] the semi-classical amplitude, i.e., the scattering amplitude with no diagrams with intermediate scalar loops, can be obtained as follows. Let us consider the

\footnotetext{
${ }^{6}$ In order to perform such a task one need to use the DBC propagator $\Delta(\tau, \sigma)=(\tau-1) \sigma \theta(\tau-\sigma)+(\sigma-$ 1) $\tau \theta(\sigma-\tau)$ alongside with the two point function of the auxiliary variables $\left\langle\lambda_{a}(\tau) \bar{\lambda}^{b}(\sigma)\right\rangle=\delta_{a}{ }^{b} \theta(\tau-\sigma)$ with $\theta(0)=1 / 2$.
} 
correlator

$$
\left\langle\Omega\left|\mathrm{T} A_{\mu}\left(x_{1}\right) A_{\nu}\left(x_{2}\right) \varphi\left(x_{1}^{\prime}\right) \varphi^{\dagger}\left(x_{2}^{\prime}\right)\right| \Omega\right\rangle=\mathcal{N}^{-1} \int \mathcal{D} g \mathcal{D} A \mathcal{D} \varphi \mathcal{D} \varphi^{*} e^{\mathrm{i} S} A_{\mu}\left(x_{1}\right) A_{\nu}\left(x_{2}\right) \varphi\left(x_{1}^{\prime}\right) \varphi^{*}\left(x_{2}^{\prime}\right),
$$

where $\mathcal{N}$ is some normalization constant and $S=S_{g}+S_{\mathrm{m}}$. Remember that the semiclassical contributions to the scattering amplitude are obtained by disregarding diagrams with scalar and graviton loops (including graviton-photon loops), which is the usual $\hbar$ power counting in path integrals. In this way, the integration over the scalar and the gauge fields generates the dressed propagators, namely

$$
\left\langle\Omega\left|\mathrm{T} A_{\mu}\left(x_{1}\right) A_{\nu}\left(x_{2}\right) \varphi\left(x_{1}^{\prime}\right) \varphi^{\dagger}\left(x_{2}^{\prime}\right)\right| \Omega\right\rangle=\tilde{\mathcal{N}}^{-1} \int \mathcal{D} g e^{\mathrm{i} S_{g}} D_{\mu \nu}\left[x_{1}, x_{2} ; g\right] G\left[x_{1}^{\prime}, x_{2}^{\prime} ; g\right] .
$$

In order to define the $S$-matrix we need the on-shell amputated propagator, which is constructed by the same prescription as in the scalar case, i.e.,

$$
D^{c}\left(p, p^{\prime}, u, \bar{u} ; g\right):=\lim _{p^{2}, p^{\prime 2} \rightarrow 0}\left(\mathrm{i} p^{2} \mathrm{i}^{\prime 2}\right) \int \mathrm{d}^{4} x_{0} \mathrm{~d}^{4} y_{0} e^{\mathrm{i} p \cdot x_{0}-\mathrm{i} p^{\prime} \cdot y_{0}} D\left(x_{0}, y_{0}, u, \bar{u} ; g\right) .
$$

The overall effect of this procedure can then again be obtained by the prescription of replacing integration region for $\tau$ from $(0, T)$ to $(-\infty, \infty)$ as in ref. [1]. The last step is to introduce physical polarization vectors, which so far have been kept track thanks to the auxiliary variables $u$ and $\bar{u}$ (see previous examples). This can be achieved by setting

$$
u_{\rho} \bar{u}_{\sigma} \rightarrow \varepsilon_{\rho}^{*}(p) \varepsilon_{\sigma}\left(p^{\prime}\right)
$$

and therefore the scattering amplitude in the semi-classical limit can be written as

$$
\begin{aligned}
\mathcal{A}(\gamma \varphi \rightarrow \gamma \varphi)= & \tilde{\mathcal{N}}^{-1} \int\left(\prod_{i=1}^{2} \mathrm{~d} x_{i} \mathrm{~d} x^{\prime}{ }_{i}\right) e^{-\mathrm{i} p_{1} x_{1}+\mathrm{i} p_{2} x_{2}} e^{-\mathrm{i} p_{1}^{\prime} x_{1}^{\prime}+\mathrm{i} p_{2}^{\prime} x_{2}^{\prime}} \\
& \times \int \mathcal{D} g e^{\mathrm{i} S_{\mathrm{EH}}} \varepsilon^{* \mu}\left(p_{1}\right) \varepsilon_{\nu}\left(p_{2}\right) D^{c}{ }_{\mu}{ }^{\nu}\left[x_{1}, x_{2} ; g\right] G^{c}\left[x_{1}^{\prime}, x_{2}^{\prime} ; g\right]
\end{aligned}
$$

where we have rewritten the dressed photon propagator in flat space-time using tetrad fields and at this point we will not distinguish between indices.

The above formula does not give yet the contributions required to study classical light bending since we still need to take consider the geometric optics regime. The reason is that to obtain the classical limit of amplitudes with spin an expansion of polarization vectors in powers of $\hbar$ is still required [49]. The relation (3.30) integrates out loops but does not produce an expansion of the polarization vectors which only appear after applying (3.29). The geometric optics regime corresponds to the situation in which we may simply replace $\varepsilon_{\nu}\left(p_{2}\right)$ by $\varepsilon_{\nu}\left(p_{1}\right)$, i.e., where the polarizations remain the same after the scattering event [47]. It is well-known that in this regime, up to factors, the scattering amplitude thus obtained is equivalent to the amplitude between one massless and one massive scalar, see e.g. [19]. Assuming this equivalence, one could build up the WQFT for a massless scalar and a massive one by taking the massless limit of one of the dressed propagators in (2.14) but this is of no interest to us. Instead we are interested in starting from first principles building up the dressed photon propagator and deriving such equivalence. In fact, this constitutes a consistency check of our setup. 


\subsection{Partition function and derivation of Feynman rules}

Once the relation to on-shell scattering amplitudes has been made the WQFT formalism instructs us to build up the following partition function

$$
\mathcal{Z}[u, \bar{u}]=\oint \frac{\mathrm{d} z}{2 \pi \mathrm{i}} \frac{e^{z \bar{u} \cdot u}}{z^{2}} \int \mathcal{D} g_{\mu \nu} \int \mathcal{D} x_{1} \int \mathcal{D} x_{2} \int D \lambda \int D \bar{\lambda} e^{\mathrm{i} S},
$$

where the full action reads

$$
S=S_{g}[g]+S_{\mathrm{p}_{1}}\left[x_{1}, \lambda, \bar{\lambda}, z ; g\right]+S_{\mathrm{p}_{2}}\left[x_{2} ; g\right],
$$

and $S_{g}$ was defined in eq. (2.13). After rescaling the worldline parameter as $\tau \rightarrow \sigma / m$ we write the scalar massive point particle action as

$$
S_{\mathrm{p}_{2}}\left[x_{2} ; g\right]=-\int_{-\infty}^{+\infty} \mathrm{d} \sigma \frac{1}{2} g_{\mu \nu} \dot{x}_{2}^{\mu} \dot{x}_{2}^{\nu}
$$

Now the point particle action can be read off from the dressed propagator of the photon in a gravitational background and gives

$$
S_{\mathrm{p}_{1}}\left[x_{1}, \bar{\lambda}, \lambda ; g\right]=-\int_{-\infty}^{+\infty} \mathrm{d} \sigma\left(\frac{1}{2} g_{\mu \nu} \dot{x}_{1}^{\mu} \dot{x}_{1}^{\nu}-\mathrm{i} \bar{\lambda} \cdot \dot{\lambda}+\frac{1}{2} \dot{x}_{1}^{\mu} \omega_{\mu}^{c d}\left(S_{c d}\right)_{a}{ }^{b} \bar{Q}^{a} Q_{b}\right)
$$

We have also rescaled the worldline parameter of the photon action

$$
\tau=\frac{\sigma}{E}
$$

introducing a parameter $E$ with dimension of energy, whose physical meaning will become clear later on. For the photon worldline, we have neglected the $R_{a}{ }^{b}$ vertex both with the counter-terms since they are sub-leading in the classical limit (see example below). Notice here that the integration regions are now from $\sigma \in(-\infty,+\infty)$ in agreement with the procedure to set dressed propagators on-shell. The partition function can now be solved perturbatively by performing wick contractions. This task can be implemented by deriving worldline Feynman rules which take care of the perturbative expansion.

Finally, we need to integrate over the modulus $z$ and consider the geometric optics regime. This regime can be applied in a similar way we did in the amplitude case using eq. (3.29) but we find more convenient to consider it directly from the partition function by defining

$$
\mathcal{Z}_{\text {geom-opt }}:=-\left.\mathcal{Z}(u, \bar{u})\right|_{\bar{u} \rightarrow u},
$$

where the minus sign is due $u \cdot \bar{u}=-1$, which is consistent with our metric signature. This definition incorporates the geometric optics limit by disregarding terms of $\mathcal{O}(\hbar)$ coming from polarization vectors as discussed at the end of last section. In addition, without loss of generality, we have chose a basis of real polarization vectors since in that limit any choice leads to the same result. 
The Feynman rules to keep track of the perturbative expansion and obtain the partition function are now easy to derive. First, performing a background expansion of the tetrads and the spin connection alongside the metric tensor we have

$$
\begin{aligned}
e_{\mu}^{a} & =\eta_{\mu}^{a}+\frac{\kappa}{2} h_{\mu}^{a}-\frac{\kappa^{2}}{8} h_{\mu \alpha} h^{\alpha a}+\mathcal{O}\left(\kappa^{3}\right), \\
\omega_{\mu}{ }^{a b} & =-\kappa \partial^{[a} h_{\mu}^{b]}-\frac{\kappa^{2}}{2} h^{\nu[a}\left(\partial^{b]} h_{\mu \nu}-\partial_{\nu} h_{\mu}^{b]}+\frac{1}{2} \partial_{\mu} h_{\nu}^{b]}\right)+\mathcal{O}\left(\kappa^{3}\right),
\end{aligned}
$$

which can be inserted in the action in (3.34) alongside with the background expansion of the configuration space variables

$$
x^{\mu}(\sigma)=b^{\mu}+p^{\mu} \sigma+q^{\mu}(\sigma)
$$

and auxiliary variables as in eq. (3.11). Notice that the momentum $p^{\mu}$ is massless for the case of the photon. The Fourier transform of the fluctuations of the auxiliary variable are given by

$$
\lambda_{a}(\sigma)=\int \hat{\mathrm{d}} \omega e^{-\mathrm{i} \omega \sigma} \lambda_{a}(\omega), \quad \bar{\lambda}^{a}(\sigma)=\int \hat{\mathrm{d}} \omega e^{\mathrm{i} \omega \sigma} \bar{\lambda}^{a}(\omega)
$$

Finally, Fourier expanding the graviton field as

$$
h_{\mu \nu}(b+p \tau+q)=\sum_{n=0}^{\infty} \frac{(-\mathrm{i})^{n}}{n !} \int \hat{\mathrm{d}}^{4} \ell e^{-\mathrm{i} \ell \cdot(b+p \tau)}(q(\tau) \cdot \ell)^{n} \varepsilon_{\mu \nu}(\ell)
$$

and plugging the above expansion in the action, we can obtain the Feynman rules associated with the photon worldline. From now on we will not distinguish between curved and tangent indices.

The 2-point functions associated with the fluctuations lead to the photon worldline propagator

$$
q^{\mu} \leadsto \stackrel{\omega}{\sim} \sim q^{\nu}=-\mathrm{i} \frac{\eta^{\mu \nu}}{2}\left(\frac{1}{(\omega+\mathrm{i} \epsilon)^{2}}+\frac{1}{(\omega-\mathrm{i} \epsilon)^{2}}\right)
$$

where, as in the case of the massive worldline, we use time-symmetric propagators. The Feynman rules associated with the photon worldline interactions read

$$
\begin{aligned}
& \left.-\mathrm{i} z k^{\alpha}\left(\eta^{\beta(\mu}\left(p^{\nu)} k_{\rho}+\omega \delta_{\rho}^{\nu)}\right)\right)\left(S_{\alpha \beta}\right)_{\lambda}{ }^{\delta} \bar{u}^{\lambda} u_{\delta}\right], \\
& =
\end{aligned}
$$

where the solid photon line represents a fluctuation of the worldline photon line. At this order we also have vertices related to the fluctuations of auxiliary variables which we obtain 
by expanding the spin connection vertex in (3.34) as well, leading to

$$
\begin{aligned}
& \text { 니 }
\end{aligned}
$$

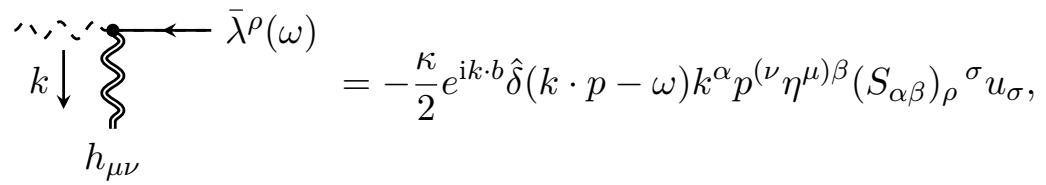

where the arrow distinguishes between $\bar{\lambda}^{a}$ and $\lambda^{a}$ which scale differently with $z$. Other rules can be easily derived, e.g., the rule for a 2 -fluctuation reads

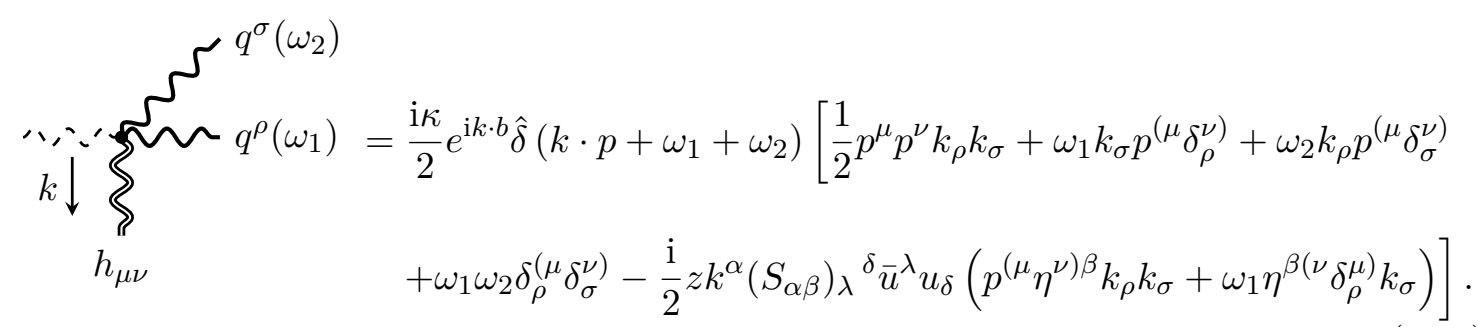

At the order we are interested we have computed all the required Feynman rules associated with the photon worldline. In addition to the rules associated with the scalar worldline in section 2 this is all we need to compute the eikonal phase.

\subsection{Definition of the eikonal phase and deflection angle}

Let us move on with the definition of the eikonal phase in the geometric optics regime. The eikonal phase is related to the partition function as

$$
\mathcal{Z}_{\text {geom-opt }}=e^{\mathrm{i} \chi}
$$

and hence by the usual definition of the eikonal phase

$$
e^{\mathrm{i} \chi}:=\frac{1}{4 m E} \int \hat{\mathrm{d}}^{4} q \hat{\delta}\left(q \cdot v_{1}\right) \hat{\delta}\left(q \cdot v_{2}\right) e^{\mathrm{i} q \cdot b} \mathcal{A}(\phi \gamma \rightarrow \phi \gamma),
$$

we can relate the scattering amplitude to the free energy of the WQFT in the geometric optics regime. Here $E$ is the energy of the photon. The rescaling we introduced in eq. (3.35) then makes the job of obtaining the eikonal phase directly in terms of momenta. We write

$$
\mathrm{i} \chi=\mathrm{i}\left(\chi_{1}+\chi_{2}+\ldots\right),
$$

where $\chi_{i}$ is of order $\mathcal{O}\left(\kappa^{2 i}\right)$. Following [24] we define the deflection angle in the small angle approximation at each order in perturbation theory by

$$
\theta_{i}=-\frac{1}{E} \frac{\partial \chi_{i}}{\partial|b|} .
$$




\subsubsection{Example}

In order to illustrate our methods let us give a simple example. The three-point amplitude $\mathcal{M}(\gamma \gamma h)$ is formally vanishing in the optical regime so let us consider its off-shell version $\mathcal{M}^{\mu \nu}(\gamma \gamma h)$ instead. Consider the 3-point vertex of (3.25) and suppose we are interested in the limit where the graviton is soft. Let $p_{2}^{\mu}=p_{1}^{\mu}+\hbar \bar{q}^{\mu}$ where $\bar{q}$ is interpreted in the classical limit as a wave-number related to the graviton. The polarization vector thus satisfies $\varepsilon_{\mu}\left(p_{2}\right)=\varepsilon_{\mu}\left(p_{1}\right)+\mathcal{O}(\hbar)$. We also parameterize the incoming photon momentum as $p_{1}=E v^{\mu}=(E, 0,0, E)$ where $E$ is the energy of the photon. Then, up to terms proportional of $\hbar$, we obtain

$$
\mathcal{M}^{\mu \nu}\left(p_{1}, p_{2}\right)=\mathrm{i} \kappa p_{1}^{\mu} p_{1}^{\nu}+\mathcal{O}(\hbar)=\mathrm{i} \kappa E^{2} v^{\mu} v^{\nu}+\mathcal{O}(\hbar)
$$

where we have used the on-shell condition on the momenta $p_{1}$ and $p_{2}$ and the transversality of the photon polarization vectors.

The equivalent object in WQFT is obtained from the LO Feynman rule (3.43). The partition function then simply reads

$$
\mathcal{Z}(z, u, \bar{u})=-\frac{\mathrm{i} \kappa}{2} \int \hat{\mathrm{d}}^{4} q e^{\mathrm{i} q \cdot b} \hat{\delta}(q \cdot p)\left[p^{\mu} p^{\nu}+2 z p^{\nu}\left(q \cdot \bar{u} u^{\mu}-\bar{u}^{\mu} q \cdot u\right)\right] .
$$

Clearly, in order to match this expression with the previous it must be that the extra terms must vanish. To see that this is the case let us consider the integration of the kernel over $z$. Exchanging the integration orders and using the identity

$$
\frac{1}{2 \pi \mathrm{i}} \oint \mathrm{d} z \frac{e^{z \bar{u} \cdot u}}{z^{k+1}}= \begin{cases}\frac{(u \cdot \bar{u})^{k}}{k !}, & k \geq 0, \\ 0, & \text { otherwise }\end{cases}
$$

we obtain

$$
\mathcal{Z}(z, u, \bar{u})=-\frac{\mathrm{i} \kappa}{2} u_{\rho} \bar{u}_{\sigma} \int \hat{\mathrm{d}}^{4} q e^{\mathrm{i} q \cdot b} \hat{\delta}(q \cdot p) p^{\mu}\left[p^{\nu} \eta^{\rho \sigma}-2 \mathrm{i} q_{\lambda}\left(S^{\lambda \nu}\right)^{\rho \sigma}\right] .
$$

Notice that the dependence on $(\bar{u}, u)$ on the first term appears due to this identity. Furthermore, using the anti-symmetry of $\left(S^{\lambda \nu}\right)_{\rho}^{\sigma}$ yields to the following relation

$$
\mathcal{Z}_{\text {geom-opt }}^{\mu \nu}=\int \hat{\mathrm{d}}^{4} q e^{\mathrm{i} q \cdot b} \hat{\delta}(q \cdot p)\left(-\frac{\mathrm{i} \kappa}{2} p^{\mu} p^{\nu}\right),
$$

where we stripped off the graviton polarization vector. Therefore, up to an irrelevant sign, we see that in the geometric optics regime the leading order Feynman rule matches the classical limit computed in eq. (3.52) as expected.

\subsection{Massless limit vs photon worldline in WQFT}

On general grounds we expect that the contributions related to the spin-terms should not contribute in the geometric optics regime since assuming the equivalence principle one may replace the photon worldline by the worldline of a massless scalar. We had a taste of this in the calculation of the lowest order partition function (3.56). For the general 


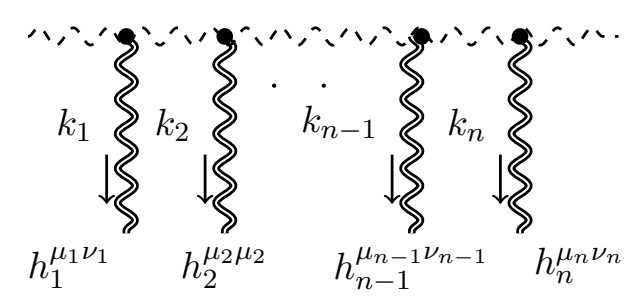

Figure 3. Dressed propagator with external photon lines on-shell.

case let us consider the diagram shown in figure 3 which appears as a sub-topology of our worldline diagrams. Let $\mathcal{K}$ denote the mathematical expression of this diagram and let $k_{i}$ be the momenta associated with each graviton line which we will consider as outgoing. The momentum of the photon worldline is labeled by $p^{\mu}$.

This diagram is still to be integrated over $z$ and hence the identity (3.54) implies that after integration all terms proportional to $z^{i}$ vanish for $i>1$. The same identity produces the factor $u \cdot \bar{u}$, which gives a non-vanishing contribution associated with the term independent of $z$. In other words only the terms independent of $z$ and proportional to $z$ contribute. Now let us rewrite the Feynman rule of a single vertex in the form

$$
V_{i}^{\mu_{1} \nu_{1}}\left(p, k_{i}\right):=\frac{\mathrm{i} \kappa}{2} e^{-\mathrm{i} k_{i} \cdot p}\left(-p^{\mu_{i}} p^{\nu_{i}}+z B_{i}^{\mu_{i} \nu_{i}}\right),
$$

where

$$
B_{i}^{\mu_{i} \nu_{i}}:=\mathrm{i} k_{i}^{\alpha} p^{\left(\nu_{i}\right.} \eta^{\left.\mu_{i}\right) \beta}\left(S_{\alpha \beta}\right)_{\rho}{ }^{\sigma} \bar{u}^{\rho} u_{\sigma} .
$$

From the Feynman rules and on the support of the Dirac delta functions this diagram can be organized as follows

$$
\mathcal{K}^{\mu_{1} \cdots \mu_{n} \nu_{1} \ldots \nu_{n}}=\frac{\mathrm{i}^{n}}{2^{n}} \kappa^{n}\left(\prod_{i=1}^{n} \hat{\delta}\left(k_{i} \cdot p\right) e^{\mathrm{i} k_{i} \cdot b}\right) K^{\mu_{1} \cdots \mu_{n} \nu_{1} \cdots \nu_{n}},
$$

where the only non-vanishing contributions are proportional to $z$ due to (3.54). Hence

$$
K^{\mu_{1} \nu_{1} \cdots \mu_{n} \nu_{n}}=(-1)^{n} p^{\mu_{1}} p^{\nu_{1}} \cdots p^{\mu_{n}} p^{\nu_{n}}-z \sum_{i=1}^{n} p^{\mu_{1}} p^{\nu_{1}} \cdots \widehat{p^{\mu_{i}} p^{\nu_{i}}} \cdots p^{\mu_{1}} p^{\nu_{1}} B_{i}^{\mu_{i} \nu_{i}}
$$

where the hat means that the factor should be excluded. Since the coefficient $B_{i}^{\mu_{i} \nu_{i}}$ depends on a single factor of the spin tensor we can use the anti-symmetry of $\left(S^{\lambda \nu}\right)_{\rho}^{\sigma}$ and that $\bar{u} \rightarrow u$ thus concluding that all terms proportional to $z$ vanish the geometric optics regime as expected. ${ }^{7}$ The same exercise can be done in the case where fluctuations on the photon worldlines or auxiliary variables are considered obtaining the same result. A simple realization of this will be shown explicitly in the next section. Let us conclude by stressing that it is only the combination of the geometric optics regime and the identity (3.54) that makes these terms vanishing. This provides an alternative path to show the equivalence between the massless limit of scattering amplitudes involving two massive particles and the

\footnotetext{
${ }^{7}$ For complex polarization vectors a similar proof can be devised.
} 
amplitudes of photons and a single massive particle in the classical limit, which in WQFT can be understood as disregarding the spin tensor.

Classical deviations from the geometric-optics regime - known as the gravitational spin Hall effect ${ }^{8}$ - have been studied from first principles in ref. [63] for the case of propagation of light and ref. [64] for propagation of gravitational waves.

\section{Calculation of deflection angles}

The WQFT setup is now complete so we are ready to apply it to perturbative calculations of the deflection angle based on the eikonal phase. An example of the calculation of the momentum impulse is shown in appendix A for the interested reader. In section 3, we have considered only the case of a spinless massive particle but it is easy to generalize it to the case of spin. The treatment of classical spinning massive particles in WQFT has been discussed at length in ref. [17] so we will build on these results. For the spinning case we will conform ourselves with a LO calculation up to quadratic order in spin.

We will use the result of section 3.5 which implies that the spin tensor plays no role in our computations. Therefore the integration over the modulus $z$ is trivial (see example in section (3.4.1)) and produces the contraction of the auxiliary variables $\bar{u} \cdot u=-1$ in the geometric optics regime. For calculations we will parametrize the momenta of the particles as $p_{1}=E v_{1}$ and $p_{2}=m v_{2}$, where $v_{1}^{2}=0$ and $v_{2}^{2}=1$. It will also be useful to choose the rest frame of the massive particle such that $v_{1}=(1,0,0,1)$ and $v_{2}=(1,0,0,0)$. Finally, recalling that $b^{\mu}$ is space-like we define $|b| \equiv \sqrt{-b^{2}}$.

\subsection{The spinless case}

Let us now move to the evaluation of the eikonal phase and the deflection angle in the spinless case. At LO there is a single diagram contributing to the eikonal phase while at NLO there are four diagrams that have the correct power counting. The latter are shown in figure 4.

\subsubsection{Leading order}

At this order the Feynman rules give a single diagram, and from our definition of the eikonal phase in section 3.4, the LO eikonal phase reads

$$
\mathrm{i} \chi_{1}=\underset{q}{q} \prod_{1}=-\mathrm{i} \kappa^{2} \frac{\left(p_{1} \cdot p_{2}\right)^{2}}{4} \int \hat{\mathrm{d}}^{4} q \hat{\delta}\left(q \cdot p_{1}\right) \hat{\delta}\left(q \cdot p_{2}\right) \frac{e^{\mathrm{i} q \cdot b}}{q^{2}} .
$$

To regulate the divergent integral in this expression we find convenient to use a cut-off regulator. The regulated integral then reads

$$
\mathcal{I}_{\succ}=\int \hat{\mathrm{d}}^{4} q \frac{\hat{\delta}\left(q \cdot p_{1}\right) \hat{\delta}\left(q \cdot p_{2}\right)}{q^{2}} e^{\mathrm{i} q \cdot b}=\frac{1}{4 \pi p_{1} \cdot p_{2}} \log \left(\frac{|b|^{2}}{L^{2}}\right) .
$$

\footnotetext{
${ }^{8}$ See ref. [62] for a review and references therein.
} 


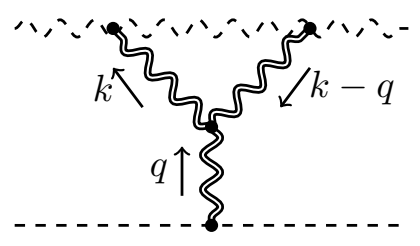

(a)

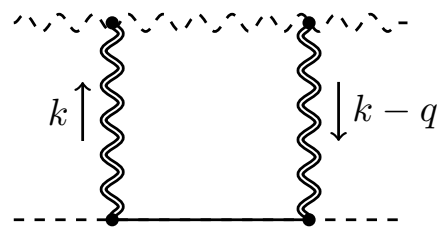

(c)

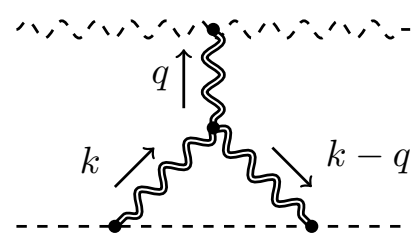

(b)

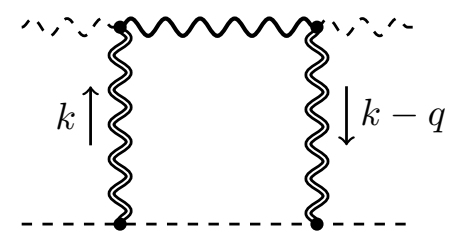

(d)

Figure 4. NLO diagrams.

To obtain this result we have set up a differential equation for $\mathcal{I}_{>} \prec$ such that the derivative produces a finite expression following similar steps as in ref. [50] with minor changes related to the parametrization of momenta. Remember that in $d=4$ we set $p_{1}=E(1,0,0,1)$ and $p_{2}=m(1,0,0,0)$. Therefore we obtain

$$
\chi_{1}=-2 G_{N}\left(p_{1} \cdot p_{2}\right) \log \left(\frac{|b|^{2}}{L^{2}}\right),
$$

where we have used $\kappa^{2}=32 \pi G_{N}$. Finally, using the rest frame of the massive particle we have

$$
\theta_{1}=-\frac{1}{E} \frac{\partial \chi_{1}}{\partial|b|}=\frac{4 G_{N} m}{|b|}
$$

which matches the result from general relativity.

\subsubsection{NLO}

Moving on with the NLO calculation, let us start with the diagrams involving the 3-graviton vertex. $^{9}$

Diagram (a). This diagram vanishes identically. In order to see this first notice that this diagram contains as a subtopology the diagram in figure 3. Therefore, from eq. (3.58) the integrand of this subtopology is proportional to

$$
\hat{\delta}\left(p_{1} \cdot q\right) \hat{\delta}\left(p_{1} \cdot k\right) p_{1}^{\mu_{1}} p_{1}^{\mu_{2}} p_{1}^{\nu_{1}} p_{1}^{\nu_{2}}
$$

Upon using the 3 -graviton vertex Feynman rule and $p_{1}^{2}=0$, we obtain identically zero due to the Dirac-delta constraints. This result is independent of the other subtopology containing the matter worldline.

\footnotetext{
${ }^{9}$ We use the conventions of ref. [65]. See also ref. [66].
} 
Diagram (b). This diagrams evaluates to

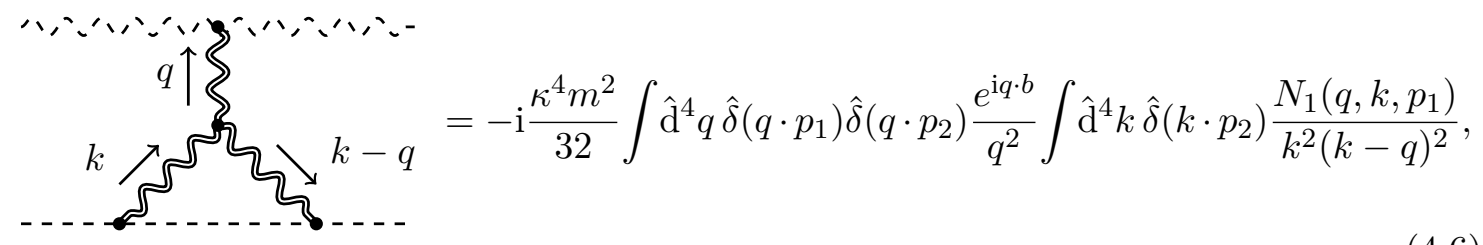

where $N_{1}\left(q, k, p_{1}\right)=\left(p_{1} \cdot p_{2}\right)^{2}\left(k^{2}+(k-q)^{2}\right)+m^{2}\left(k \cdot p_{1}\right)^{2}$. The terms multiplying $\left(p_{1} \cdot p_{2}\right)^{2}$ lead to tadpole integrals which are vanishing. Using our parametrization of momenta let us focus on the integral

$$
I_{1}=\int \hat{\mathrm{d}}^{4} k \hat{\delta}\left(k \cdot v_{2}\right) \frac{\left(k \cdot v_{1}\right)^{2}}{k^{2}(k-q)^{2}}=v_{1}^{\mu} v_{1}^{\nu} \int \hat{\mathrm{d}}^{4} k \hat{\delta}\left(k \cdot v_{2}\right) \frac{k_{\mu} k_{\nu}}{k^{2}(k-q)^{2}} .
$$

On the support of the Dirac-delta constraints $\hat{\delta}\left(q \cdot v_{1}\right) \hat{\delta}\left(q \cdot v_{2}\right)$ this integral can be reduced by performing a simple Passarino-Veltman reduction leading to

$$
I_{1}=\frac{v_{1}^{\mu} v_{1}^{\nu}}{8}\left(3 q_{\mu} q_{\nu}+q^{2}\left(v_{2 \mu} v_{2 \nu}-\eta_{\mu \nu}\right)\right) \int \hat{\mathrm{d}}^{4} k \frac{\hat{\delta}\left(k \cdot v_{2}\right)}{k^{2}(k-q)^{2}}=\frac{q^{2} \sigma^{2}}{8} \int \hat{\mathrm{d}}^{4} k \frac{\hat{\delta}\left(k \cdot v_{2}\right)}{k^{2}(k-q)^{2}}
$$

where we have defined $\sigma=v_{1} \cdot v_{2}$ to write the result in a Lorentz invariant form. Then we are left to calculate the integral

$$
I_{\triangleright}:=\int \hat{\mathrm{d}}^{4} q \hat{\delta}\left(q \cdot v_{1}\right) \hat{\delta}\left(q \cdot v_{2}\right) e^{\mathrm{i} q \cdot b} \int \hat{\mathrm{d}}^{4} k \frac{\hat{\delta}\left(k \cdot v_{2}\right)}{k^{2}(k-q)^{2}},
$$

which can be computed following ref. [27] adapted to our case. The result is

$$
I_{\triangleright}=\frac{1}{16 \pi|b|} .
$$

Let us now move on to diagrams with the topology of a box.

Diagram (c). This diagram evaluates to

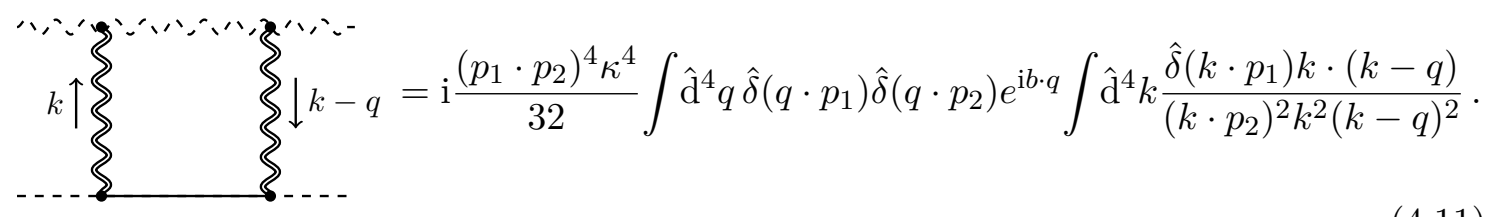

The integral reduction produces integrals with double poles on the same side of the complex plane. Following ref. [67] these integrals vanish after closing the contour in the opposite direction. ${ }^{10}$

\footnotetext{
${ }^{10}$ For time symmetric propagators one simple applies this argument twice for each i $\epsilon$ prescription.
} 


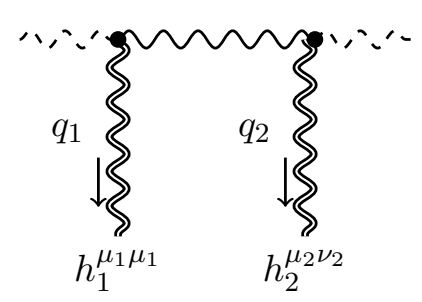

Figure 5. Subtopology of diagram with photon fluctuation.

Diagram (d). Let us show that in this case the spin tensor does not contribute in the geometric optics regime. For that consider a simpler version of the exercise in section 3.5 but now including a single fluctuation as shown in figure 5. Focusing on the terms proportional to $z$ we find that the integrand is proportional to the tensor structures

$$
\mathrm{i} \eta^{\mu_{1} \nu_{2}} p_{1}^{\mu_{2}}\left(S_{\alpha}^{\nu_{1}}\right)^{\rho \sigma} u_{\rho} \bar{u}_{\sigma} q_{i}^{\alpha}, \quad \mathrm{i} p_{1}{ }^{\mu_{1}} p_{1}{ }^{\nu_{1}} q_{i}{ }^{\nu_{2}}\left(S_{\alpha}^{\mu_{2}}\right)^{\rho \sigma} u_{\rho} \bar{u}_{\sigma} q_{i}^{\alpha}, \quad i=1,2,
$$

which vanish in the geometric optics regime. The other contributions simplify to

$$
\text { l } l k-q=\mathrm{i} \frac{\kappa^{4}\left(p_{1} \cdot p_{2}\right)^{2}}{32} \int \hat{\mathrm{d}}^{4} q \hat{\delta}\left(q \cdot p_{1}\right) \hat{\delta}\left(q \cdot p_{2}\right) e^{\mathrm{i} q \cdot b} \int \hat{\mathrm{d}}^{4} k \frac{\hat{\delta}\left(k \cdot p_{2}\right) N_{2}\left(q, k, p_{1}\right)}{\left(k \cdot p_{1}\right)^{2} k^{2}(k-q)^{2}}
$$

where $N_{2}=\left(p_{1} \cdot p_{2}\right)^{2}\left(k^{2}-k \cdot q\right)+2 m^{2}\left(k \cdot p_{1}\right)^{2}$. The integral reduction produces finite integrals with double poles on the same side of the complex plane which we can set to zero. Therefore the only surviving term is the one that cancels the double, which is proportional to $(4.9)$.

Therefore after adding up the contributing diagrams (b) and (d) the result of the eikonal phase reads

$$
\chi_{2}=\kappa^{4} \frac{15}{256} m\left(p_{1} \cdot p_{2}\right) \frac{1}{16 \pi|b|},
$$

and using our parametrization of momenta the scattering angle reads

$$
\theta_{2}=-\frac{1}{E} \frac{\partial \chi_{2}}{\partial|b|}=\frac{15 \pi}{4} \frac{G_{N}^{2} m^{2}}{|b|^{2}}
$$

in agreement with the massless limit of the scattering angle of two massive objects in which one of the masses goes to zero.

\subsection{Spinning massive particle}

The case of a spinning massive particle can be treated along the same lines. The description in ref. [17] is based on the inclusion of supersymmetry at the level of the worldline action. The WQFT thus constructed is valid at quadratic order in spin. Here we conform ourselves 
with summarizing the LO Feynman rule for this case. Performing the same rescaling as in the previous section we have the Feynman rule

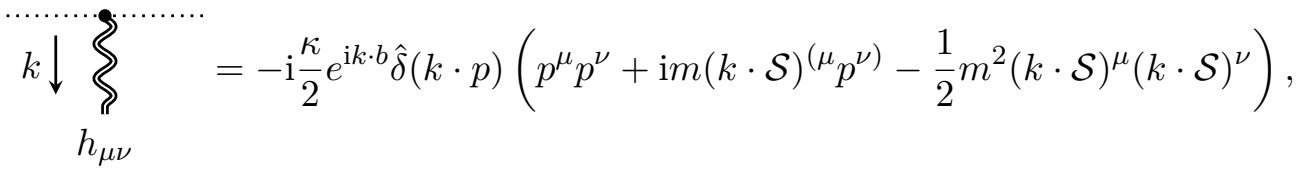

where $(k \cdot \mathcal{S})^{\mu}:=k_{\nu} \mathcal{S}^{\nu \mu}$. Therefore at LO the eikonal phase is computed from a single diagram obtaining

$$
\mathrm{i} \chi_{1}=\underset{1}{ }=-\mathrm{i} \kappa^{2} \frac{\left(p_{1} \cdot p_{2}\right)^{2}}{4} \int \hat{\mathrm{d}}^{4} q \hat{\delta}\left(q \cdot p_{1}\right) \hat{\delta}\left(q \cdot p_{2}\right) \frac{e^{\mathrm{i} q \cdot b}\left(1+N_{\mathcal{S}}\right)}{q^{2}},
$$

with the numerator $N_{\mathcal{S}}$ is given by

$$
N_{\mathcal{S}}=-\frac{\mathrm{i} m}{p_{1} \cdot p_{2}}\left(p_{1} \cdot \mathcal{S} \cdot q\right)-\frac{m^{2}}{2\left(p_{1} \cdot p_{2}\right)^{2}}\left(p_{1} \cdot \mathcal{S} \cdot q\right)^{2} .
$$

Specializing the spin tensor defined in ref. [17] for the case at hand, let us parametrize it as

$$
\mathcal{S}^{\mu \nu}=\frac{2 s}{|b|}\left(b^{[\mu}\left(v_{1}-\sigma v_{2}\right)^{\nu]}\right) .
$$

Hence, after reintroducing the dimensionless velocities, we can rewrite the numerator as

$$
N_{\mathcal{S}}=-\mathrm{i} s \frac{b \cdot q}{|b|}-s^{2} \frac{1}{2|b|^{2}}(b \cdot q)^{2} .
$$

To complete the calculation we need to evaluate the following type of integrals

$$
\mathcal{I}^{\mu_{1} \mu_{2} \ldots \mu_{n}}:=\int \hat{\mathrm{d}}^{4} q \hat{\delta}\left(q \cdot v_{1}\right) \hat{\delta}\left(q \cdot v_{2}\right) e^{\mathrm{i} q \cdot b} \frac{q^{\mu_{1}} \cdots q^{\mu_{n}}}{q^{2}} .
$$

The simplest case $\mathcal{I}^{\mu_{1}}$ can be computed from (4.2). For the case $\mathcal{I}^{\mu_{1} \mu_{2}}$ we adapt the procedure of ref. [49] to our case. Noting that the results must lie in the plane orthogonal to the four velocities the integral $\mathcal{I}^{\mu_{1} \mu_{2}}$ can be written as

$$
\mathcal{I}^{\mu_{1} \mu_{2}}=c_{1} b^{\mu_{1}} b^{\mu_{2}}+c_{2} \Pi^{\mu_{1} \mu_{2}},
$$

where $\Pi^{\mu_{1} \mu_{2}}$ is a projector which explicitly reads

$$
\Pi^{\mu_{1} \mu_{2}}=\eta^{\mu_{1} \mu_{2}}+v_{1}^{\mu_{1}} v_{1}^{\mu_{2}}-v_{2}^{\mu_{1}} v_{1}^{\mu_{2}}-v_{1}^{\mu_{1}} v_{2}^{\mu_{2}}
$$

Therefore employing the traceless property of $\mathcal{I}^{\mu_{1} \mu_{2}}$ and (4.2) we obtain

$$
\mathcal{I}^{\mu_{1} \mu_{2}}=\frac{1}{\pi b^{4} \sigma}\left(b^{\mu_{1}} b^{\mu_{2}}-\frac{1}{2} b^{2} \Pi^{\mu_{1} \mu_{2}}\right) .
$$


After some algebra we compute

$$
\chi_{1}=\kappa^{2} \frac{p_{1} \cdot p_{2}}{8 \pi}\left(-\frac{1}{2} \log \left(\frac{\left|b^{2}\right|}{L^{2}}\right)-\frac{s}{|b|}+\frac{s^{2}}{2|b|^{2}}\right),
$$

which leads to the scattering angle

$$
\theta_{1}=4\left(\frac{1}{|b|}-\frac{s}{|b|^{2}}+\frac{s^{2}}{|b|^{3}}\right) G_{N} m
$$

in agreement with the massless limit of the Kerr-result of ref. [17] (see also refs. [68, 69]).

\section{Conclusions}

We have extended the worldline quantum field theory formalism to classical observables to the case of scattering of light off a massive particle. We have constructed in a worldline representation the gravitationally dressed photon propagator. Then, following the WQFT formalism, we have identified the partition function and derived the Feynman rules to compute the eikonal phase and the deflection angle from it.

The dressed photon propagator is built from a matrix-valued particle action whose path integral requires time ordering. To get rid of the latter, we have introduced auxiliary variables that at the same time describe explicitly the spin degrees of freedom. They play an important role at the quantum level to obtain the correct amplitudes as we have seen in section 3.2. However, time ordering is a quantum requirement and hence the geometric optics regime makes the terms related to the spin-tensor vanish, thus providing an alternative realization of the equivalence between the amplitude of two massive particles and that of a massive particle and a massless. This simplifies the calculation of the eikonal phase dramatically and only two diagrams are required to calculate the NLO contribution. We have computed the NLO scattering deflection angle for spinless and LO for spinning particles, finding full agreement with the results by other methods. Our calculation is compatible with the eikonal approach based on on-shell scattering amplitudes.

An alternative approach to construct a worldline representation of the photon propagator would be to consider the $N=2$ spinning particle, which has been used to describe the quantum theory of spin 1 and differential forms in refs. [57, 58, 70,71]. Indeed, in ref. [17] this model has been used to describe spinning black holes. However, the approach we have used here is closer to the standard QFT setup. It would be interesting to compare against the massless limit of the purely worldline approach of ref. [17] based on the $N=2$ spinning particle.

Our dressed propagator can also be applied to the description of light by light scattering, and it would be interesting to study amplitudes made up of dressed photon propagators in the case where the spin tensor contributes. Another interesting route would be to explore the so-called generalized Wilson line [72-75] for photons. Dressed photon propagators are also useful in describing the propagation of light in an arbitrary medium [76] so it would be interesting to consider coupling to matter in addition to gravity. In this case, the geometricoptics regime is not applicable and terms associated with the spin-tensor are expected to contribute. 


\section{Acknowledgments}

LDLC acknowledges financial support from the Open Physics Hub at the Physics and Astronomy Department "Augusto Righi" in Bologna.

\section{A Next to leading order photon impulse}

In the WQFT one can define the observables related to the point particle mechanics, by making use of Noether theorem which in such case is nothing else but Ehrenfest theorem. Let us first define the impulse of the photon as [1]

$$
\Delta p^{\mu}=\int_{-\infty}^{+\infty} \mathrm{d} \sigma\left\langle\frac{\mathrm{d}^{2} q^{\mu}}{\mathrm{d} \sigma^{2}}\right\rangle=\int_{-\infty}^{+\infty} \hat{\mathrm{d}} \omega\left(-\omega^{2}\left\langle\tilde{q}^{\mu}(\omega) \hat{\delta}(\omega)\right\rangle\right)
$$

The leading order calculation is straightforward and can be obtained by the evaluation of a single diagram which, unlike the eikonal phase, has two kinematic fluctuations, namely

$$
\Delta^{(0)} p^{\mu}=\underset{1}{2}=-\kappa^{2} \frac{\left(p_{1} \cdot p_{2}\right)^{2}}{4} \frac{\partial I_{\succ}}{\partial b_{\mu}}=4 G_{N}\left(p_{1} \cdot p_{2}\right) \frac{b^{\mu}}{|b|^{2}},
$$

where the tree level integral $I_{\succ} \prec$ has been evaluated in (4.2).

Let us move now to the next to leading order photon impulse. The topologies which are vanishing for the eikonal phase do also vanish here in the same way. Particularly, the diagram with the three-graviton vertex with the two gravitons starting from the photon line is exactly zero once using momentum conservation and the delta constraints. Thus, the only non-vanishing contributions arise from

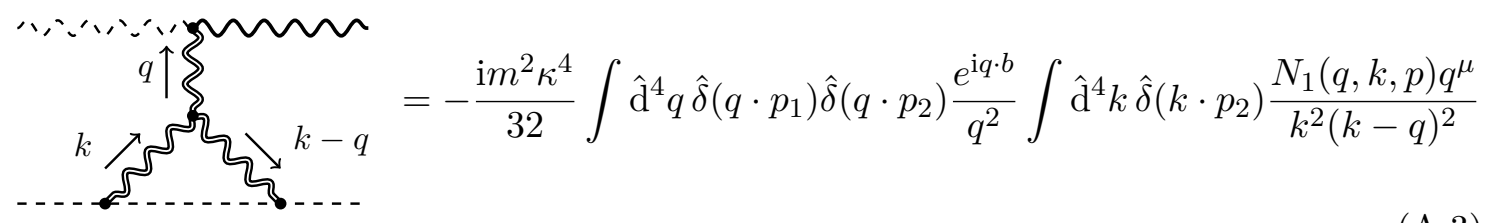

with the numerator $N_{1}=\left(p_{1} \cdot p_{2}\right)^{2}\left(k^{2}+(k-q)^{2}\right)+m^{2}\left(k \cdot p_{1}\right)^{2}$, and the other contributing diagram is

$$
\text { } \int \hat{\mathrm{d}}^{4} k \hat{\delta}\left(k \cdot p_{2}\right) \frac{N_{2}(q, k, p)(q-k)^{\mu}}{k^{2}(k-q)^{2}\left(k \cdot p_{1}\right)^{2}},
$$

where $N_{2}=\left(p_{1} \cdot p_{2}\right)^{2} k \cdot(k-q)+2 m^{2}\left(k \cdot p_{1}\right)^{2}$. Let us briefly review the integration procedure. We first focus on eq. (A.3). For this one we just need to evaluate the integral

$$
\mathcal{I}_{1}=\int \hat{\mathrm{d}}^{4} q \hat{\delta}\left(q \cdot v_{1}\right) \hat{\delta}\left(q \cdot v_{2}\right) \frac{e^{\mathrm{i} q \cdot b}}{q^{2}} \int \hat{\mathrm{d}}^{4} k \hat{\delta}\left(k \cdot v_{2}\right) \frac{\left(k \cdot v_{1}\right)^{2}}{k^{2}(k-q)^{2}}=\frac{1}{128 \pi|b|}
$$


since we can rewrite the whole expression in the r.h.s. of eq. (A.3) as

$$
-\frac{\mathrm{i} E m^{2} \kappa^{4}}{32} \frac{1}{\mathrm{i}} \frac{\partial}{\partial b^{\mu}} \mathcal{I}_{1}=-\frac{1}{4} \pi G_{N}^{2} m\left(p_{1} \cdot p_{2}\right) \frac{b^{\mu}}{|b|^{3}} .
$$

Let us move now to the next diagram, which can be recast as follows

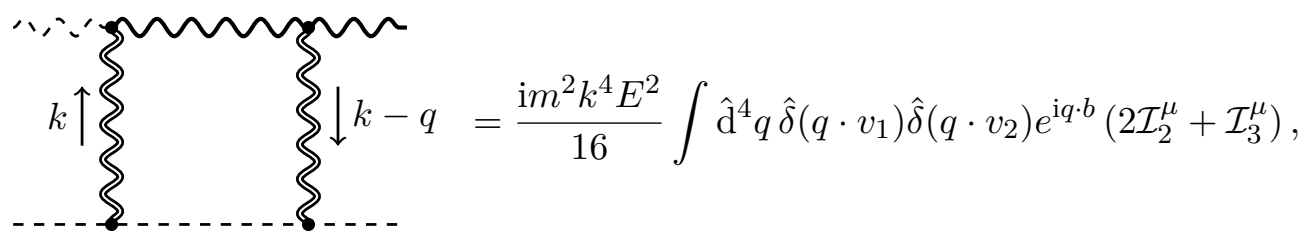

where

$$
\mathcal{I}_{2}^{\mu}=\int \hat{\mathrm{d}}^{4} k \frac{\hat{\delta}\left(k \cdot v_{2}\right)(q-k)^{\mu}}{k^{2}(q-k)^{2}}, \quad \mathcal{I}_{3}^{\mu}=\int \hat{\mathrm{d}}^{4} k \frac{\hat{\delta}\left(k \cdot v_{2}\right) k \cdot(k-q)(q-k)^{\mu}}{k^{2}(q-k)^{2}\left(k \cdot v_{1}\right)^{2}} .
$$

Let us perform the tensor reduction of the above integrals. The first one can be decomposed as $\mathcal{I}_{2}^{\mu}=A q^{\mu}+B v_{2}^{\mu}$ such that, using the delta constraint $q \cdot v_{1}=q \cdot v_{2}=0$, one finds that $B=0$. Contracting with $q^{\mu}$ one finds that

$$
A=\frac{1}{2} \int \hat{\mathrm{d}}^{4} k \frac{\hat{\delta}\left(k \cdot v_{2}\right)}{k^{2}(k-q)^{2}} .
$$

In this way one is able to evaluate the first contribution in (A.7) as

$$
\frac{\mathrm{i} m^{2} \kappa^{4} E}{8} \int \hat{\mathrm{d}}^{4} q \hat{\delta}\left(q \cdot v_{1}\right) \hat{\delta}\left(q \cdot v_{2}\right) e^{\mathrm{i} q \cdot b} \mathcal{I}_{2}^{\mu}=4 \pi G_{N}^{2} m\left(p_{1} \cdot p_{2}\right) \frac{b^{\mu}}{|b|^{3}} .
$$

Before proceeding further, let us point out that adding up the two contributions just evaluated i.e. (A.6) and (A.10) one obtains

$$
\frac{15 \pi}{4} G_{N}^{2} m\left(p_{1} \cdot p_{2}\right) \frac{b^{\mu}}{|b|^{3}},
$$

which corresponds to the impulse obtained from the NLO eikonal phase, without any iteration of the scattering data. However, we still need to evaluate the first piece in (A.7). For this task we write

$$
\mathcal{I}_{3}^{\mu}=A q^{\mu}+B v_{2}^{\mu}+C v_{1}^{\mu} .
$$

Then, using that $v_{2}^{\mu} M_{\mu}=0$ one obtains $B=-\sigma C$, which allows us to rewrite $\mathcal{I}_{3}^{\mu}=$ $A q^{\mu}+C\left(v_{1}^{\mu}-\sigma v_{2}^{\mu}\right)$. In this way, after contracting with $q^{\mu}$, one finds that the integral thus obtained are tadpoles therefore concluding that $A=0$. Contracting with $v_{1}^{\mu}$ and using integral reduction allows us to fix the remaining coefficient, namely

$$
C=\frac{1}{2} q^{2} \int \hat{\mathrm{d}}^{4} k \frac{\hat{\delta}\left(k \cdot v_{2}\right)}{\left(k^{2}+\mathrm{i} \epsilon\right)\left((k-q)^{2}+\mathrm{i} \epsilon\right)\left(k \cdot v_{1}+\mathrm{i} \epsilon\right)}=-\frac{\mathrm{i}}{2} q^{2} \int \hat{\mathrm{d}}^{4} k \frac{\hat{\delta}\left(k \cdot v_{1}\right) \hat{\delta}\left(k \cdot v_{2}\right)}{k^{2}(k-q)^{2}},
$$

where we performed the change of variables $k-q \rightarrow-k^{\prime}$ in the last equality and the Dirac delta representation

$$
\hat{\delta}(x)=\mathrm{i}\left(\frac{1}{x+\mathrm{i} \epsilon}-\frac{1}{x-\mathrm{i} \epsilon}\right)
$$


which enables us to rewrite the full contribution related to $\mathcal{I}_{3}$ as the square of $I_{\succ} \prec$ which has been evaluated previously, see eq. (4.2).

Finally, putting all pieces together and performing the extra momentum integration, one obtains the next to leading order photon impulse

$$
\Delta p^{\mu}=4 G_{N}\left(p_{1} \cdot p_{2}\right) \frac{b^{\mu}}{|b|^{2}}+G_{N}^{2} m \frac{\left(p_{1} \cdot p_{2}\right)}{|b|}\left(\frac{15 \pi}{4} \frac{b^{\mu}}{|b|^{2}}-\frac{8}{|b|}\left(v_{1}^{\mu}-\sigma v_{2}^{\mu}\right)\right)
$$

where we reintroduced $\sigma=v_{1} \cdot v_{2}$ to write the result in a Lorentz invariant form.

Open Access. This article is distributed under the terms of the Creative Commons Attribution License (CC-BY 4.0), which permits any use, distribution and reproduction in any medium, provided the original author(s) and source are credited.

\section{References}

[1] G. Mogull, J. Plefka and J. Steinhoff, Classical black hole scattering from a worldline quantum field theory, JHEP 02 (2021) 048 [arXiv:2010.02865] [INSPIRE].

[2] K. Daikouji, M. Shino and Y. Sumino, Bern-Kosower rule for scalar QED, Phys. Rev. D 53 (1996) 4598 [hep-ph/9508377] [INSPIRE].

[3] N. Ahmadiniaz, A. Bashir and C. Schubert, Multiphoton amplitudes and generalized Landau-Khalatnikov-Fradkin transformation in scalar QED, Phys. Rev. D 93 (2016) 045023 [arXiv: 1511.05087] [INSPIRE].

[4] N. Ahmadiniaz, F. Bastianelli and O. Corradini, Dressed scalar propagator in a non-Abelian background from the worldline formalism, Phys. Rev. D 93 (2016) 025035 [Addendum ibid. 93 (2016) 049904] [arXiv: 1508.05144] [INSPIRE].

[5] J.P. Edwards and C. Schubert, One-particle reducible contribution to the one-loop scalar propagator in a constant field, Nucl. Phys. B 923 (2017) 339 [arXiv:1704.00482] [INSPIRE].

[6] N. Ahmadiniaz, F. Bastianelli, O. Corradini, J.P. Edwards and C. Schubert, One-particle reducible contribution to the one-loop spinor propagator in a constant field, Nucl. Phys. B 924 (2017) 377 [arXiv: 1704.05040] [INSPIRE].

[7] N. Ahmadiniaz, V.M. Banda Guzmán, F. Bastianelli, O. Corradini, J.P. Edwards and C. Schubert, Worldline master formulas for the dressed electron propagator. Part I. Off-shell amplitudes, JHEP 08 (2020) 049 [arXiv: 2004.01391] [INSPIRE].

[8] O. Corradini and G.D. Esposti, Dressed Dirac propagator from a locally supersymmetric $N=1$ spinning particle, Nucl. Phys. B 970 (2021) 115498 [arXiv:2008.03114] [InSPIRE].

[9] N. Ahmadiniaz, V.M.B. Guzman, F. Bastianelli, O. Corradini, J.P. Edwards and C. Schubert, Worldline master formulas for the dressed electron propagator. Part 2. On-shell amplitudes, JHEP 01 (2022) 050 [arXiv:2107.00199] [INSPIRE].

[10] E. Fradkin, Application of functional methods in quantum field theory and quantum statistics (II), Nucl. Phys. 76 (1966) 588 [inSPIRE].

[11] M. Fabbrichesi, R. Pettorino, G. Veneziano and G.A. Vilkovisky, Planckian energy scattering and surface terms in the gravitational action, Nucl. Phys. B 419 (1994) 147 [hep-th/9309037] [INSPIRE]. 
[12] W.D. Goldberger and I.Z. Rothstein, An Effective field theory of gravity for extended objects, Phys. Rev. D 73 (2006) 104029 [hep-th/0409156] [INSPIRE].

[13] W.D. Goldberger and I.Z. Rothstein, Towers of Gravitational Theories, Gen. Rel. Grav. 38 (2006) 1537 [hep-th/0605238] [INSPIRE].

[14] W.D. Goldberger and A. Ross, Gravitational radiative corrections from effective field theory, Phys. Rev. D 81 (2010) 124015 [arXiv:0912.4254] [INSPIRE].

[15] G.U. Jakobsen, G. Mogull, J. Plefka and J. Steinhoff, Classical Gravitational Bremsstrahlung from a Worldline Quantum Field Theory, Phys. Rev. Lett. 126 (2021) 201103 [arXiv:2101.12688] [INSPIRE].

[16] G.U. Jakobsen, G. Mogull, J. Plefka and J. Steinhoff, Gravitational Bremsstrahlung and Hidden Supersymmetry of Spinning Bodies, Phys. Rev. Lett. 128 (2022) 011101 [arXiv:2106.10256] [INSPIRE].

[17] G.U. Jakobsen, G. Mogull, J. Plefka and J. Steinhoff, SUSY in the sky with gravitons, JHEP 01 (2022) 027 [arXiv:2109.04465] [INSPIRE].

[18] C. Shi and J. Plefka, Classical double copy of worldline quantum field theory, Phys. Rev. D 105 (2022) 026007 [arXiv:2109.10345] [INSPIRE].

[19] N.E.J. Bjerrum-Bohr, B.R. Holstein, J.F. Donoghue, L. Planté and P. Vanhove, Illuminating Light Bending, PoS CORFU2016 (2017) 077 [arXiv: 1704.01624] [INSPIRE].

[20] J.F. Donoghue, General relativity as an effective field theory: The leading quantum corrections, Phys. Rev. D 50 (1994) 3874 [gr-qc/9405057] [INSPIRE].

[21] N.E.J. Bjerrum-Bohr, J.F. Donoghue and B.R. Holstein, Quantum gravitational corrections to the nonrelativistic scattering potential of two masses, Phys. Rev. D 67 (2003) 084033 [Erratum ibid. 71 (2005) 069903] [hep-th/0211072] [INSPIRE].

[22] N.E.J. Bjerrum-Bohr, J.F. Donoghue and P. Vanhove, On-shell Techniques and Universal Results in Quantum Gravity, JHEP 02 (2014) 111 [arXiv:1309.0804] [INSPIRE].

[23] N.E.J. Bjerrum-Bohr, J.F. Donoghue, B.R. Holstein, L. Planté and P. Vanhove, Bending of Light in Quantum Gravity, Phys. Rev. Lett. 114 (2015) 061301 [arXiv:1410.7590] [InSPIRE].

[24] N.E.J. Bjerrum-Bohr, J.F. Donoghue, B.R. Holstein, L. Plante and P. Vanhove, Light-like Scattering in Quantum Gravity, JHEP 11 (2016) 117 [arXiv:1609.07477] [INSPIRE].

[25] D. Bai and Y. Huang, More on the Bending of Light in Quantum Gravity, Phys. Rev. D 95 (2017) 064045 [arXiv: 1612.07629] [InSPIRE].

[26] N.E.J. Bjerrum-Bohr, J.F. Donoghue, B.K. El-Menoufi, B.R. Holstein, L. Planté and P. Vanhove, The Equivalence Principle in a Quantum World, Int. J. Mod. Phys. D 24 (2015) 1544013 [arXiv: 1505.04974] [INSPIRE].

[27] N.E.J. Bjerrum-Bohr, P.H. Damgaard, G. Festuccia, L. Planté and P. Vanhove, General Relativity from Scattering Amplitudes, Phys. Rev. Lett. 121 (2018) 171601 [arXiv: 1806. 04920] [INSPIRE].

[28] D. Amati, M. Ciafaloni and G. Veneziano, Higher Order Gravitational Deflection and Soft Bremsstrahlung in Planckian Energy Superstring Collisions, Nucl. Phys. B 347 (1990) 550 [INSPIRE].

[29] S. Melville, S.G. Naculich, H.J. Schnitzer and C.D. White, Wilson line approach to gravity in the high energy limit, Phys. Rev. D 89 (2014) 025009 [arXiv:1306.6019] [InSPIRE]. 
[30] A. Luna, S. Melville, S.G. Naculich and C.D. White, Next-to-soft corrections to high energy scattering in QCD and gravity, JHEP 01 (2017) 052 [arXiv:1611.02172] [INSPIRE].

[31] R. Akhoury, R. Saotome and G. Sterman, High Energy Scattering in Perturbative Quantum Gravity at Next to Leading Power, Phys. Rev. D 103 (2021) 064036 [arXiv:1308.5204] [INSPIRE].

[32] A. Koemans Collado, P. Di Vecchia and R. Russo, Revisiting the second post-Minkowskian eikonal and the dynamics of binary black holes, Phys. Rev. D 100 (2019) 066028 [arXiv: 1904.02667] [INSPIRE].

[33] A. Cristofoli, P.H. Damgaard, P. Di Vecchia and C. Heissenberg, Second-order Post-Minkowskian scattering in arbitrary dimensions, JHEP 07 (2020) 122 [arXiv: 2003.10274] [INSPIRE].

[34] P. Di Vecchia, A. Luna, S.G. Naculich, R. Russo, G. Veneziano and C.D. White, A tale of two exponentiations in $\mathcal{N}=8$ supergravity, Phys. Lett. B 798 (2019) 134927 [arXiv: 1908.05603] [INSPIRE].

[35] P. Di Vecchia, S.G. Naculich, R. Russo, G. Veneziano and C.D. White, A tale of two exponentiations in $\mathcal{N}=8$ supergravity at subleading level, JHEP 03 (2020) 173 [arXiv: 1911.11716] [INSPIRE].

[36] Z. Bern, H. Ita, J. Parra-Martinez and M.S. Ruf, Universality in the classical limit of massless gravitational scattering, Phys. Rev. Lett. 125 (2020) 031601 [arXiv:2002.02459] [INSPIRE].

[37] J. Parra-Martinez, M.S. Ruf and M. Zeng, Extremal black hole scattering at $\mathcal{O}\left(G^{3}\right)$ : graviton dominance, eikonal exponentiation, and differential equations, JHEP 11 (2020) 023 [arXiv: 2005. 04236] [INSPIRE].

[38] P. Di Vecchia, C. Heissenberg, R. Russo and G. Veneziano, The eikonal approach to gravitational scattering and radiation at $\mathcal{O}\left(G^{3}\right)$, JHEP 07 (2021) 169 [arXiv:2104.03256] [INSPIRE].

[39] C. Heissenberg, Infrared divergences and the eikonal exponentiation, Phys. Rev. D 104 (2021) 046016 [arXiv:2105.04594] [INSPIRE].

[40] P.H. Damgaard, L. Plante and P. Vanhove, On an exponential representation of the gravitational S-matrix, JHEP 11 (2021) 213 [arXiv:2107.12891] [INSPIRE].

[41] L. de la Cruz, A. Luna and T. Scheopner, Yang-Mills observables: from KMOC to eikonal through EFT, JHEP 01 (2022) 045 [arXiv:2108.02178] [INSPIRE].

[42] Z. Bern, A. Luna, R. Roiban, C.-H. Shen and M. Zeng, Spinning black hole binary dynamics, scattering amplitudes, and effective field theory, Phys. Rev. D 104 (2021) 065014 [arXiv: 2005. 03071] [INSPIRE].

[43] M. Accettulli Huber, A. Brandhuber, S. De Angelis and G. Travaglini, Eikonal phase matrix, deflection angle and time delay in effective field theories of gravity, Phys. Rev. D 102 (2020) 046014 [arXiv: 2006. 02375] [INSPIRE].

[44] D. Kosmopoulos and A. Luna, Quadratic-in-spin Hamiltonian at $\mathcal{O}\left(G^{2}\right)$ from scattering amplitudes, JHEP 07 (2021) 037 [arXiv: 2102.10137] [INSPIRE].

[45] A. Brandhuber, G. Chen, G. Travaglini and C. Wen, Classical gravitational scattering from a gauge-invariant double copy, JHEP 10 (2021) 118 [arXiv:2108.04216] [INSPIRE]. 
[46] R. Aoude and A. Ochirov, Classical observables from coherent-spin amplitudes, JHEP 10 (2021) 008 [arXiv : 2108.01649] [INSPIRE].

[47] A. Cristofoli, R. Gonzo, D.A. Kosower and D. O'Connell, Waveforms from Amplitudes, arXiv:2107.10193 [INSPIRE].

[48] D.A. Kosower, B. Maybee and D. O'Connell, Amplitudes, Observables, and Classical Scattering, JHEP 02 (2019) 137 [arXiv:1811.10950] [inSPIRE].

[49] B. Maybee, D. O'Connell and J. Vines, Observables and amplitudes for spinning particles and black holes, JHEP 12 (2019) 156 [arXiv:1906.09260] [INSPIRE].

[50] L. de la Cruz, B. Maybee, D. O'Connell and A. Ross, Classical Yang-Mills observables from amplitudes, JHEP 12 (2020) 076 [arXiv:2009.03842] [INSPIRE].

[51] F. Bastianelli and P. van Nieuwenhuizen, Path integrals and anomalies in curved space, Cambridge Monographs on Mathematical Physics Cambridge University Press, Cambridge U.K. (2006).

[52] F. Bastianelli, R. Bonezzi, O. Corradini and E. Latini, Extended SUSY quantum mechanics: transition amplitudes and path integrals, JHEP 06 (2011) 023 [arXiv:1103.3993] [INSPIRE].

[53] F. Bastianelli and A. Zirotti, Worldline formalism in a gravitational background, Nucl. Phys. B 642 (2002) 372 [hep-th/0205182] [INSPIRE].

[54] C. Schubert, Perturbative quantum field theory in the string inspired formalism, Phys. Rept. 355 (2001) 73 [hep-th/0101036] [INSPIRE].

[55] D. Amati, M. Ciafaloni and G. Veneziano, Superstring Collisions at Planckian Energies, Phys. Lett. B 197 (1987) 81 [INSPIRE].

[56] F. Bastianelli and P. van Nieuwenhuizen, Trace anomalies from quantum mechanics, Nucl. Phys. B 389 (1993) 53 [hep-th/9208059] [INSPIRE].

[57] F. Bastianelli, P. Benincasa and S. Giombi, Worldline approach to vector and antisymmetric tensor fields, JHEP 04 (2005) 010 [hep-th/0503155] [INSPIRE].

[58] F. Bastianelli, P. Benincasa and S. Giombi, Worldline approach to vector and antisymmetric tensor fields. II, JHEP 10 (2005) 114 [hep-th/0510010] [INSPIRE].

[59] F. Bastianelli, R. Bonezzi, O. Corradini and E. Latini, Particles with non abelian charges, JHEP 10 (2013) 098 [arXiv: 1309.1608] [InSPIRE].

[60] F. Bastianelli, F. Comberiati and L. de la Cruz, Worldline description of a bi-adjoint scalar and the zeroth copy, JHEP 12 (2021) 023 [arXiv:2107.10130] [INSPIRE].

[61] F. Bastianelli and R. Bonezzi, One-loop quantum gravity from a worldline viewpoint, JHEP 07 (2013) 016 [arXiv: 1304.7135] [INSPIRE].

[62] M.A. Oancea, C.F. Paganini, J. Joudioux and L. Andersson, An overview of the gravitational spin Hall effect, arXiv: 1904.09963 [INSPIRE].

[63] M.A. Oancea, J. Joudioux, I.Y. Dodin, D.E. Ruiz, C.F. Paganini and L. Andersson, Gravitational spin Hall effect of light, Phys. Rev. D 102 (2020) 024075 [arXiv:2003.04553] [INSPIRE].

[64] L. Andersson, J. Joudioux, M.A. Oancea and A. Raj, Propagation of polarized gravitational waves, Phys. Rev. D 103 (2021) 044053 [arXiv:2012.08363] [InSPIRE]. 
[65] N.E.J. Bjerrum-Bohr, B.R. Holstein, L. Planté and P. Vanhove, Graviton-Photon Scattering, Phys. Rev. D 91 (2015) 064008 [arXiv: 1410.4148] [InSPIRE].

[66] J. Plefka, J. Steinhoff and W. Wormsbecher, Effective action of dilaton gravity as the classical double copy of Yang-Mills theory, Phys. Rev. D 99 (2019) 024021 [arXiv: 1807.09859] [INSPIRE].

[67] G. Kälin and R.A. Porto, Post-Minkowskian Effective Field Theory for Conservative Binary Dynamics, JHEP 11 (2020) 106 [arXiv:2006.01184] [INSPIRE].

[68] T. Ono, A. Ishihara and H. Asada, Gravitomagnetic bending angle of light with finite-distance corrections in stationary axisymmetric spacetimes, Phys. Rev. D 96 (2017) 104037 [arXiv: 1704.05615] [INSPIRE].

[69] R. Kumar, B.P. Singh and S.G. Ghosh, Shadow and deflection angle of rotating black hole in asymptotically safe gravity, Annals Phys. 420 (2020) 168252 [arXiv: 1904.07652] [InSPIRE].

[70] F. Bastianelli and R. Bonezzi, Quantum theory of massless (p,0)-forms, JHEP 09 (2011) 018 [arXiv:1107.3661] [INSPIRE].

[71] F. Bastianelli, R. Bonezzi and C. Iazeolla, Quantum theories of (p,q)-forms, JHEP 08 (2012) 045 [arXiv: 1204 .5954] [INSPIRE].

[72] E. Laenen, G. Stavenga and C.D. White, Path integral approach to eikonal and next-to-eikonal exponentiation, JHEP 03 (2009) 054 [arXiv: 0811.2067] [INSPIRE].

[73] C.D. White, Factorization Properties of Soft Graviton Amplitudes, JHEP 05 (2011) 060 [arXiv: 1103.2981] [INSPIRE].

[74] D. Bonocore, A. Kulesza and J. Pirsch, Classical and quantum gravitational scattering with Generalized Wilson Lines, arXiv:2112.02009 [INSPIRE].

[75] D. Bonocore, Asymptotic dynamics on the worldline for spinning particles, JHEP 02 (2021) 007 [arXiv: 2009.07863] [INSPIRE].

[76] M. Difallah, A. Szameit and M. Ornigotti, Path-integral description of quantum nonlinear optics in arbitrary media, Phys. Rev. A 100 (2019) 053845 [arXiv:1904.02548] [INSPIRE]. 